\title{
The Bric-à-Brac transcription factors are necessary for formation of functional germline stem cell niches through control of $d p p$ expression in the Drosophila melanogaster ovary
}

\begin{abstract}
Many studies have focused on the mechanisms of stem cell maintenance via their interaction with a particular niche or microenvironment in adult tissues, but how formation of a functional niche is initiated, including how stem cells within a niche are established, is less well understood. Adult Drosophila melanogaster ovary Germline Stem Cell (GSC) niches are comprised of somatic cells forming a stack called a Terminal Filament (TF) and underlying Cap Cells (CCs) and Escort Cells (ECs), which are in direct contact with GSCs. In the adult, the Engrailed (En) transcription factor is specifically expressed in niche cells where it directly controls expression of the decapentaplegic gene $(d p p)$ encoding a member of the Bone Morphogenetic Protein (BMP) family of secreted signaling molecules, which are key factors for GSC maintenance. In late third instar larval ovaries, in response to BMP signaling from newly-formed niches, adjacent primordial germ cells become GSCs. The bric-àbrac paralogs (bab1 and bab2) encode BTB/POZ-domain containing transcription factors, that are also expressed in developing GSCs niches where they are required for TF formation. Here, we demonstrate that Bab1 and Bab2 display redundant cell autonomous function for TF morphogenesis and we identify a new function for these genes in GSC establishment. Moreover, we show that Bab proteins control $d p p$ expression in otherwise correctly specified CCs, independently of En and its paralog Invected (Inv). In fact, our results also indicate that en/inv function in larval stages are neither essential for TF formation, nor GSC establishment. Finally, when bab2 was overexpressed in ovarian somatic cells outside of the niche, where en/inv were not expressed, ectopic BMP signaling activation was induced in adjacent germ cells of adult ovaries, which formed GSC-like tumors. Together, these results indicate that Bab transcription factors are positive regulators of BMP signaling for acquisition of GSC status.
\end{abstract}




\section{INTRODUCTION}

A stem cell niche or microenvironment allows, first, the establishment of stem cells, and second, the maintenance of a balance between stem cell self-renewal and differentiation. Much more is known about stem cell maintenance than about initial stem cell establishment. The interactions between niche and stem cells need to be strictly controlled for maintaining homeostasis of adult tissues. In fact, a defect in stem cell homeostasis can be pathological in humans, producing for example unregulated cancer stem cells (Plaks et al., 2015; Prager et al., 2019; Zhao et al., 2018), and may also be an important part of normal and premature aging when stem cell populations lose their potential to selfrenew (Ermolaeva et al., 2018). The discovery of pre-metastatic niches in cancer (Aguado et al., 2017; Kaplan et al., 2005) also makes the study of the properties of stem cell niches a key for gaining headway in cancer biology.

The Drosophila melanogaster adult ovary has proven to be an excellent model for understanding how interaction with adjacent somatic niche cells allows for maintenance of Germline Stem Cell (GSC) status (Greenspan et al., 2015; Xie and Spradling, 1998). Approximately 20 individual GSC niches, each associated with a small number (2-3) of GSCs, are present in the Drosophila adult ovary at the tip of structures called germaria (Fig 1A). Each GSC niche is composed of several types of somatic cells: a Terminal Filament (TF), which is a stack of about 8 flattened cells, approximately 5 Cap Cells (CCS) present at the base of the TF (Gilboa, 2015), one triangularly-shaped transition cell connecting the TF and CCs (Panchal et al., 2017) and posterior to CCs, the anterior Escort Cells (ECs) (Fig 1A) (Wang and Page-McCaw, 2018). Both CCs and anterior ECs, are in direct contact with GSCs. CCs are considered to be the key component of GSC niches, their number correlating closely with the number of GSCs (Song et al., 2007; Xie and Spradling, 2000). CCs anchor GSCs to the niche by DE-cadherin-mediated adhesion (Song et al., 2002) and produce two Bone Morphogenetic Proteins (BMPs), Decapentaplegic (Dpp) and Glass Bottom Boat (Gbb), acting as short-range secreted signals required for GSC maintenance (Liu et al., 2010, 2015; Luo et al., 2017; Song et al., 2004; Wang et al., 2008; Xie and Spradling, 1998, 2000). Indeed, $\mathrm{Dpp} / \mathrm{Gbb}$ signal is transduced in GSCs, via the presence of a specific receptor composed of both Thickveins and Punt, leading to phosphorylation of the transcription factor Mad (pMad), translocation of pMad into the nucleus and transcriptional repression of germline 
differentiation genes such as bag-of-marbles (bam) (Chen and McKearin, 2003b; Song et al., 2004). It was shown that the homeobox transcription factor Engrailed (En) binds $d p p$ cisregulatory sequences in vitro and activates $d p p$ transcription in CCs (Luo et al., 2017). Several other signaling pathways, such as the JAK/STAT (Lopez-Onieva et al., 2008; Wang et al., 2008) and Hedgehog (Liu et al., 2015; Lu et al., 2015; Rojas-Ríos et al., 2012) pathways, are active in niche cells and have also been implicated in regulation of $d p p$ expression in these cells. In addition, the ectopic expression of $d p p$ or engrailed and ectopic activation of JAK/STAT signaling in somatic cells of the germarium, as well as the loss-of-function of bam in germ cells, all lead to a germarial GSC tumorous phenotype further supporting the implication of these factors in GCS homeostasis (Eliazer et al., 2014; McKearin and Ohlstein, 1995; Song et al., 2004; Wang et al., 2008; Xie and Spradling, 1998).

Morphogenesis of GSC niches is a highly stereotyped process occurring in the third instar larval ovary. Morphogenesis begins with TF formation which involves flattening, sorting, intercalation and stacking of somatic TF cell precursors, initiating at the medial side of the ovary and progressing as a wave laterally (Fig 1A) (Godt and Laski, 1995; SahutBarnola et al., 1996). Individualization of each TF is accomplished by the migration of apical somatic cells (Inner Sheath Cells) between TFs (Fig 1A) (Cohen et al., 2002).The number of TFs that form in the larval ovary determines the number of GSC niches at the adult stage (Bartoletti et al., 2012; Green et al., 2011; Sarikaya et al., 2012). At the base of each newlyformed TF, the anterior-most Intermingled Cells (ICs-somatic cells intermingled with Primordial Germ Cells, PGCs) differentiate into CCs adopting a cuboidal shape clearly distinguishable from that of the flat TF cells (Fig $1 \mathrm{~A}$ and Fig $1 \mathrm{~B}$ and $\mathrm{B}^{\prime}$, yellow and green brackets, respectively) (Panchal et al., 2017; Zhu and Xie, 2003). At this stage, the transition cell is already distinguishable (Fig $1 \mathrm{~A}$ and 1B', green arrowhead) (Panchal et al., 2017). Posterior-most ICs will give rise to ECs in adult ovaries (Fig 1A) (Lai et al., 2017).

Very few genes have been reported to be implicated in TF formation, notably, the bric-à-brac locus paralogs (bab1/bab2) (Bartoletti et al., 2012; Couderc et al., 2002; Godt and Laski, 1995; Green and Extavour, 2012; Sahut-Barnola et al., 1996) and the engrailed/invected locus paralogs (en/inv) (Bolívar et al., 2006; Gustavson et al., 1996). The bab1 and bab2 genes encode proteins sharing evolutionarily conserved domains: a BTB (Broad-Complex, Tramtrack and Bric-à-brac)/POZ (POx virus and Zinc finger) domain involved 
in homodimeric and heterodimeric interactions (Chaharbakhshi and Jemc, 2016) and a BabConserved Domain (BabCD) containing two known motifs, a Pipsqueak (Psq) domain and an AT-hook like motif both involved in DNA-protein interactions (Couderc et al., 2002). In the larval ovary, loss of function alleles of the bab locus lead to a dominant phenotype characterized by an excess of TFs, resulting in an excess of GSC niches in adults (Bartoletti et al., 2012), and by a recessive phenotype characterized by a defect in TF formation resulting in atrophied ovaries with few germ cells in adults and sterility (Godt and Laski, 1995; SahutBarnola et al., 1995). As for the engrailed/invected locus, induction of clones homozygous for a deletion encompassing both genes identified a cell autonomous function for these genes in the correct alignment of TF cells during TF formation (Bolívar et al., 2006). CC specification has been shown to require the combined action of Notch signaling and the large Maf transcription factor Traffic Jam (Tj) (Gancz et al., 2011; Hsu and Drummond-Barbosa, 2011; Panchal et al., 2017; Song et al., 2007; Yatsenko and Shcherbata, 2018).

The newly-formed niches can be considered functional at the prepupal stage when the underlying PGCs become GSCs, that is, when they begin to undergo asymmetric divisions producing two different types of daughter cells, a GSC that remains within the niche and a cystoblast that will give rise to a germline cyst (Zhu and Xie, 2003). Before niche formation, that is, before the mid third instar larval stage, all PGCs have been shown to exhibit BMP signaling activation (Gilboa and Lehmann, 2004; Kai and Spradling, 2004), and this is correlated with detection of Dpp in all somatic cells of the ovary (Sato et al., 2010). During the larval to pupal stages, Dpp signaling prevents PGC differentiation into germline cysts (Gilboa and Lehmann, 2004; Matsuoka et al., 2013; Zhu and Xie, 2003) and promotes PGC proliferation (Sato et al., 2010; Zhu and Xie, 2003). Upon niche formation, dpp expression (Matsuoka et al., 2013; Zhu and Xie, 2003) and Dpp protein accumulation (Sato et al., 2010), become unevenly distributed in the ovary by an as yet uncharacterized mechanism, with highest levels found in niche cells. Among GCs, only those in contact with the Dpp-secreting niche cells retain BMP signaling activation as evidenced by the presence of pMad (Fig 1C and $1 C^{\prime \prime}$, red and pink arrowheads) (Gancz et al., 2011) and become GSCs that retain the capacity to divide (Zhu and Xie, 2003). The more posterior PGCs, not in contact with niche cells, lose Dpp signaling activation (Fig 1C-1C", white arrowheads) and proceed to differentiate into cystoblasts, whereupon they produce the first germline cysts (Matsuoka et al., 2013; Tseng et al., 2018; Zhu and Xie, 2003). 
In this study, we addressed the role of $b a b 1$ and $b a b 2$ in formation of functional GSC niches, in particular, on establishment of the first GSCs in the larval ovary. In developing ovaries, Bab1 has been reported to be present only in niche cell and Bab2 in all somatic cells, however at higher levels in niche cells (Couderc et al., 2002). We also asked whether the two $b a b$ genes have distinct or redundant functions within developing niche cells for either niche formation or GSC establishment. For this, we used a null allele of bab1 (bab ${ }^{\text {A128) }}$ ) and Gal4RNAi targeted knockdown of each or both of the two paralogs specifically in GSC niches during their formation in larvae. This approach has allowed us to show that bab1 and bab2 display redundant cell autonomous functions for TF formation. In contrast, cells exhibiting several CC characteristics were nonetheless present upon depletion of Bab proteins in niches. In addition, we have identified a new essential role for bab genes in prepupal niches for initial establishment of GSCs correlated with a role in dpp expression in CCs. The function of $b a b$ in CCs is unlikely to require that of the en/inv genes since their expression levels are not affected when Bab1 and Bab2 are depleted. Unexpectedly, we also found that En/Inv are not essential for activation of BMP signaling and GSC establishment in the larval ovary, contrasting with their essential functions in adult ovaries for BMP-mediated GSC maintenance. Finally, when bab2 was overexpressed in somatic ovarian cells as of larval stages, GSC-like tumorous germaria were produced in the adult ovary. This was associated with ectopic BMP signaling activation occurring in GCs without the presence of En/Inv in the bab2-overexpressing somatic ovarian cells. Together, our results indicate that the bab genes as necessary for $d p p$ expression in the newly-forming GSC niches and for establishment of the first GSCs.

\section{RESULTS}

\section{bab1 and bab2 share redundant cell autonomous functions for TF morphogenesis}

We addressed the effect of depletion of Bab1 and Bab2 proteins, either separately or together, specifically in two niche cell populations, TF cells and CCs, during niche formation at the third instar larval stage. First, we tested and validated two strategies based on the use of RNAi and/or mutant alleles to deplete each of these two proteins specifically in TFs and CCs. Bab2 is detected in all ovarian somatic cells at this stage, but at higher levels in TFs and CCs as previously reported (Couderc et al., 2002) (Fig 1D, green and yellow brackets, 
respectively). We used a hedgehog-Gal4 ( $h h G)$ driver in order to express a bab2-specific RNAi construct $\left(U A S-b a b 2^{I R}\right)$ only in niche cells during their differentiation (Lai et al., 2017; Sarikaya and Extavour, 2015). In parallel, hhG was also used to drive a UAS-GFP reporter construct ( $h h G>G F P$ ) to mark niche cells (thereafter named $h h G+$ cells). In control $h h G>G F P$ prepupal ovaries, hhG driver expression was found to be specific to niche TF cells and CCS, with much higher GFP accumulation in medial vs. lateral niches (Fig $2 \mathrm{~A}$ and $2 \mathrm{~A}^{\prime}$, yellow vs. blue dotted lines, respectively, and 2A"). Inside each niche, $h$ hG>GFP expression levels were mosaic between TF cells and always low in CCs (Fig 2A'). Consistent with the expression profile of the $h h G$ driver (Fig 2B' and 2B"), in $h h G>U A S-b a b 2^{I R}$ ovaries, Bab2 depletion was observed in all medial TF cells, but not in all CCs (Fig 2B', yellow dotted lines), and was not observed in lateral $h h G+$ cells (Fig 2B', blue dotted lines). Noteworthily, in niche cells knocked down for bab2, the level of Bab1 was not noticeably affected (Fig 2B'). Bab1 accumulation in the control hhG>GFP ovaries was detected at high levels in both TF cells and CCs as reported (Couderc et al., 2002) (Fig 1D', green and yellow brackets, respectively). In addition, low level of Bab1 were also detected in ICs (Fig 1D', blue bracket). For bab1 depletion, we used $b a b^{A 128}$, reported as a null mutant allele of bab1 not affecting bab2 expression (Couderc et al., 2002; Godt et al., 1993). Indeed, in prepupal ovaries from females homozygous for $b a b^{A 128}$, bab1 expression was undetectable, whereas the level of Bab2 did not appear to be different from that in the $h h G>G F P$ control (Fig $2 \mathrm{C}$ and $C^{\prime}$ ).

In either the $h h G>U A S-b a b 2^{I R}$ or the homozygous $b a b^{A 128}$ context, use of $h h G>G F P$ to mark TFs indicated that the morphology of TFs and TF cells (Fig $2 \mathrm{~B}$ and $2 \mathrm{~B}^{\prime}$ and $2 \mathrm{C}$ and $2 \mathrm{C}^{\prime}$ ), the number of TFs per ovary (Fig 2F) and that of TF cells per TF (Fig 2G) were not different from that in the control. This was also the case when a UAS-dicer2 construct was used to increase the effect of the UAS-bab2 $2^{I R}$ construct (Fig $3 C-3 C^{\prime \prime}$ ). From these results, we concluded that loss of function of bab1 alone or a strong depletion of Bab2 protein in TF cells did not affect TF morphogenesis.

Next, $b a b 1$ and $b a b 2$ were knocked down together, in the $b a b^{A 128}, h h G>G F P, b a b 2^{I R}$ or in the $h h G>G F P, b a b 1^{1 R}, b a b 2^{I R}$ contexts. In $b a b^{A 128}, h h G>G F P, b a b 2^{I R}$ prepupal ovaries (Fig 2D), Bab1 protein was absent from all $h h G+$ cells (Fig 2D', yellow and blue dotted lines, respectively) and ICs as expected in bab ${ }^{A 128}$ mutants. In the same ovaries, Bab2 protein was efficiently depleted in medial but not lateral $h h G+$ cells (Fig 2D', yellow vs. blue dotted lines). In $h h G>G F P, b a b 1^{I R}, b a b 2^{I R}$ ovaries (Fig $2 \mathrm{E}$ ), bab1 and bab2 were only efficiently knocked 
down in medial $h h G+$ cells, but not in lateral $h h G+$ cells (Fig $2 E^{\prime}$, yellow vs. blue dotted line, respectively). In both genetic contexts, the medial part of the ovary contained a large cluster of $h h G+$ cells, which, unlike in the control, failed to form TFs (Fig 2D' and 2E', yellow dotted line). These results are consistent with the previously-described phenotypes of bab locus mutants (Godt and Laski, 1995). We quantified the number of TFs per ovary (Fig 2F), the flattening of the medial $h h G+$ cells (Fig $2 \mathrm{H}$ ) and found that these parameters were significantly lower than those in the control, while the overall number of $h h G+$ cells per ovary was the same as that in the control (Fig 2I). In contrast, in the lateral-most part of the ovary, where $h h G$ driver expression was lower and incomplete depletion of Bab proteins was obtained (Fig 2D-D" and 2E-E"), hhG + cells formed TFs that were not different from control lateral TFs (Fig 2D' and 2E' compared to $2 \mathrm{~A}^{\prime}$, blue dotted lines). The higher penetrance of the abnormally low TF number per ovary phenotype using $b a b^{A 128}$ instead of UAS-bab1 $1^{1 R}$ for the double $b a b 1 / b a b 2$ knockdown (Fig 2F) may be attributable to the more efficient depletion obtained for Bab1 with $b a b^{A 128}$ than with $U A S-b a b 1^{I R}$ (Fig 2D' compared to 2E'). Since reduction of both Bab1 and Bab2 in niche cells impaired TF formation significantly, while reduction of each one individually did not, we conclude that bab1 and bab2 share redundant, cell autonomous functions for TF morphogenesis.

The functions of bab1 and bab2 are necessary in niche cells for GSC establishment in larval ovaries

As shown above, although $h h G+$ cells depleted of Bab1 and Bab2 do not form morphologically normal niches, the normal number of $h h G+$ cells are present as a mass of cells in the medial part of the ovary. We asked whether this un-organized $h h G+$ cell mass was able to recruit the initial GSCs in the prepupal ovary. The same approaches as above were used to deplete Bab1 and Bab2 proteins from niche cells, either separately or together. To test for functional niche activity, BMP pathway activation in GCs was used as a characteristic of GSC identity at this stage. For this, accumulation of a direct downstream component of BMP pathway activation, phosphorylated Mad (pMad), was monitored in GCs marked by the presence of Vasa. In control prepupal ovaries, pMad was detected in anterior-most GCs adjacent to niches at either high or medium levels (Fig $1 C^{\prime}$ and $1 C^{\prime \prime}$, red and pink arrowheads, respectively, and $3 A$ and $\left.3 A^{\prime}\right)$. In addition, pMad was also detected in two somatic cell populations, in particular, at a low level in CCs and at an intermediate level 
in peripheral posterior cells (Fig $1 \mathrm{C}^{\prime}$ and $1 \mathrm{C}^{\prime \prime}$, yellow and blue arrowheads, respectively and Fig 3A'and 3B', white arrowheads, respectively). This is consistent with a previous report indicating BMP signaling activation in somatic cells using a different reporter (Zhu and Xie, 2003). In a bab1 homozygous null mutant background ( $b a b^{A 128}$, hhG>GFP), pMad+ GSCS were present at the base of TFs (Fig $3 B$ and $3 B^{\prime}$ ) and in the same number as that in the control (Fig 3F). In $h$ hG>GFP, dicer2, bab2 $2^{/ R}$ ovaries (Fig 3C), very rare niches containing only CCs with undetectable levels of Bab2 were obtained (Fig $3 C^{\prime}$ and $C^{\prime \prime}$, yellow dotted line). However, in these cases, GCs that were adjacent to Bab2-depleted CC groups (yellow dotted line) were always positive for pMad (Figure $3 C^{\prime}$ and $3 C^{\prime \prime}$ ). These results suggest that the depletion of either Bab1 or Bab2 individually in niche cells did not affect GSC establishment.

Upon strong depletion of both Bab1 and Bab2 in either bab ${ }^{A 128}$, hhG> GFP $b a b 2^{1 R}$ or $h h G>G F P, b a b 1^{I R}, b a b 2^{I R}$ ovaries, Vasa+ GCs were found in close contact with the medial hhG+ cells, but almost all GCs were devoid of pMad staining (Fig 3D and 3D' and 3E and 3E', below yellow dotted line). In contrast, in the lateral region where TFs were formed, $\mathrm{pMad}^{+}$ GCs (i.e. GSCs) were found at the base of each niche as observed in control ovaries (Fig 3D and $3 \mathrm{D}^{\prime}$, and Fig $3 \mathrm{E}$ and $3 \mathrm{E}^{\prime}$, bellow blue dotted lines). The overall number of $\mathrm{pMad}^{+} \mathrm{GCs}$ per ovary was significantly reduced when compared to that in the control and, when present, these GSCs were associated with lateral TFs in almost all of cases (Fig 3F). Thus, in absence of both bab1 and bab2 functions, GCs were present and correctly positioned next to $h$ h $G+$ cells, but the BMP pathway was, for the most part, not activated in these cells, indicating that GSC status was compromised. We next asked whether these GCs may differentiate precociously. Germline cell differentiation can be monitored by the expression of a GFP transcriptional reporter for bam expression (bamP-GFP) (Chen and McKearin, 2003a). In control prepupal ovaries (bamP-GFP, $h$ hG>lacZ), GCs (GFP+/Vasa+) that differente were only rarely detected among GCs contacting $h h G>U A S-l a c Z$ ( $\beta$-Gal+) niche cells, whereas all GCs located one cell diameter away from these niche cells expressed this differentiation marker (Fig 4A and 4A', Vasa+ cells under encircled TFs, and Fig 4C). In contrast, differentiating GCs (GFP+/Vasa+) were found significantly more frequently in direct contact with clusters of disorganized $h h G>U A S-l a c Z$ ( $\beta$-Gal+) cells depleted of Bab1 and Bab2 in the medial zone of prepupal ovaries than in control ovaries (Fig $4 \mathrm{~B}$ and 4B', arrowhead, and Fig 4C). Abnormal differentiation of some GCs adjacent to the cluster of medial $h h G+$ cells thus correlated with the abnormal absence of the GSC marker pMad in the same region. Taken together these 
results indicate that $b a b$ function is required in niches for acquisition of GSC status by PGCs as marked by sustained activation of the BMP pathway and absence of expression of the differentiation reporter bamP-GFP. Our results also indicate that Bab1 and Bab2 depletion in $h h G+$ cells does not hinder GCs from remaining in close contact with $h h G+$ cells, indicating that the absence of BMP pathway activation in these GCs cannot be attributed to an incapacity of these cells to contact niche cells. Together, our work strongly suggest that bab1 and bab2 share redundant functions for GSC establishment in the late larval ovary.

\section{The functions of bab1 and bab2 are not required in niche cells for expression of Cap Cell specification markers}

CCs have been shown to be essential for GSC establishment and maintenance (Panchal et al., 2017; Song et al., 2007; Ward et al., 2006; Xie and Spradling, 2000). Our results here show that Bab proteins are necessary in niche cells of the larval ovary for GSC establishment. One hypothesis is that bab function is necessary for specification of CCS which in turn would recruit the initial GSCs among PGCs. In order to test this possibility, we characterized the nature of the $h h G+$ cells upon Bab1 and Bab2 depletion using a combination of markers that allow the distinction to be made between TF cells and CCs in prepupal niches: Traffic Jam (Tj) (Li et al., 2003), P1444-LacZ (Panchal et al., 2017), Delta (Panchal et al., 2017; Song et al., 2007) and E(spl)mb-CD2 (a transcriptional reporter of Notch activity) (de Celis et al., 1998).

The first two markers tested were $\mathrm{Tj}$ and P1444-lacZ. In prepupal control hhG+ niches, $t j$ and P1444-LacZ were expressed at high levels in CC nuclei (Fig 5A-5A", yellow bracket) and sometimes also in transition cells (the basal-most TF cell) (Fig 5A'-A"' and Fig $5 C^{\prime}$, green arrowheads), at a low level for $t j$, consistent with what was previously reported (Panchal et al., 2017). P1444-LacZ was also expressed in some TF cells at a much lower level than in CCS and in transition cells (Fig 5A-5A", green bracket). Therefore, within niches, high levels of $t j$ and P1444-lacZ expression designates CCs, while low levels or absence of P1444lacZ expression without any Tj distinguishes TF cells. Upon depletion of Bab1 and Bab2 by RNAi specifically in niche cell precursors, the disorganized clustered medial $h h G+$ cells were composed of two cell populations. One of the populations was posteriorly-positioned, adjacent to Vasa+ GCs, and presented Tj nuclear accumulation, comparable to that detected in control CCs (Fig 5B' and 5B'"' and Fig 5D', yellow brackets), associated with the expression 
of P1444-lacZ, albeit at a lower level than in control CCs (Fig 5B'-5B', yellow bracket). Therefore, in posterior $h h G+$ cells, these CC markers are present in the absence of Bab proteins. The second population of cells within the medial $h h G+$ cell cluster positioned more anteriorly, like control TF cells, did not accumulate Tj (Fig 5B' and 5D', green brackets), but unlike control TF cells, did not express even low levels of P1444-lacZ (Fig 5B'-5B', green bracket). The identity of this second population may thus be mixed and this could be linked to the inability of these cells to form proper TFs.

We next tested for the presence of Notch pathway-associated niche markers, i.e. the Notch ligand Delta and a Notch transcriptional reporter, E(spl)mb-CD2. It has been shown that during niche morphogenesis, CC specification depends on Notch signaling (Hsu and Drummond-Barbosa, 2011; Song et al., 2007). More recently, it was shown that the transition cell was associated primarily with vesicular Delta, designating an active Delta signal-sending state (Yatsenko and Shcherbata, 2018). Consequent activation of Notch signaling in adjacent ICs was proposed to allow their specification into CCs. In $h$ hG>GFP control prepupal ovaries, TF cells presented high levels of Delta at the plasma membrane (Fig 5C-5C', green bracket), transition cells were associated with active Delta within cytoplasmic vesicles (Fig 5C-5C', green arrowhead), and CCs were largely devoid of Delta except in few vesicles (Fig 5C-5C', yellow bracket and white arrow), consistent with previous reports (Panchal et al., 2017; Yatsenko and Shcherbata, 2018). CD2 from the E(spl)mB-CD2 reporter was detected at the plasma membrane of both TF cells and CCS (Fig 5E-5E", green and yellow brackets, respectively), with highest levels in posterior-most TF cells. In anteriormost $h h G+$ cells knocked down for bab1 and bab2, Delta was detected only in its active cytoplasmic vesicular form (Fig 5D' and 5D', green brackets and arrows), and not at the plasma membrane, unlike in control TF cells (compare to $5 \mathrm{C}^{\prime}$ and $5 \mathrm{C}^{\prime \prime}$, green brackets), while posterior-most $h h G+/ T j+$ cells did not present any Delta at the plasma membrane nor in vesicles as for most of the control CC population (compare yellow brackets in Fig 5D' and $5 D^{\prime \prime}$ to Fig $5 C^{\prime}$ and $\left.5 C^{\prime \prime}\right)$. Despite this, all $h h G+$ cells depleted of Bab1 and Bab2 expressed $E(s p l) m 6-C D 2$ reporter indicative of active Notch signaling (Fig 5F' and $5 \mathrm{~F}^{\prime \prime}$, green and yellow brackets), as observed in control niche cells. Therefore, depletion of Bab proteins in niche cells did not prevent Notch signaling activation in these cells. This is likely due to vesicular Delta present in the more anterior Tj-/hhG + cells, since at the end of the third instar larval stage, the most posterior TF cells become Delta-sending cells presenting predominantly 
vesicular Delta and activating Notch signaling in adjacent CC precursors (Yatsenko and Shcherbata, 2018). Altogether, these results show that bab1 and bab2 are not necessary for several aspects of CC specification during niche formation since posterior-most $h h G+$ cells depleted of Bab proteins present the appropriate expression pattern for all CC markers tested. Despite the presence of CC-marker expressing cells upon Bab depletion in niches, Vasa+ GCs in contact with these CCs are not GSCs since they are not activated for BMP signaling as evidenced by the absence of nuclear pMad (Fig 5B'" compare to Fig 5A"', arrowheads). Therefore, the absence of GSC establishment with Bab-depleted hhG+ cells cannot be attributed to failure in CC specification as far as the markers tested here.

\section{$b a b 1$ and $b a b 2$ are necessary for dpp transcription in niche cells at the time of initial GSC establishment}

Since Bab proteins do not seem to be necessary for CC specification in the larval ovary, the absence of GSC establishment at this stage upon depletion of these transcription factors in niche cells could be due to a specific defect in dpp expression in CCs. Therefore, we tested whether the bab locus function controls $d p p$ expression in CCs. For this we used two lines of the GFP-tagged FlyFos TransgeneOme library (Sarov et al., 2016), hereafter called $d p p-G F P$ and $d p p-n / s G F P$. Each line contains a construction with a large genomic region (about $44 \mathrm{~kb}$ ) covering both the $d p p$ regulatory and coding sequences into which a Cterminal GFP tag was introduced. We first characterized the adult ovarian expression profile of these two lines. In adult germaria (Fig S1), we found that the two fusion proteins were detected at high levels in CCs (S1A'", $\mathrm{B}^{\prime \prime \prime}$ Fig, yellow brackets), sometimes in ECs (S1B Fig, blue brackets) and absent from TF cells ( $S 1 A^{\prime \prime \prime}, B^{\prime \prime \prime}$ Fig, green brackets), and were also detected in prefollicular cells (S1B Fig, orange brackets), reflecting the endogenous $d p p$ expression pattern (Liu et al., 2010, 2015; Luo et al., 2017; Wang et al., 2008; Xie and Spradling, 2000). For analysis in prepupal ovaries, we used the dpp-nlsGFP transgene since the nuclear Dpp:GFP fusion protein produced was less likely to perturb endogenous Dpp function. Indeed, no obvious alteration of wild type phenotype was observed in ovaries of females carrying this transgene (Fig 6A). At the prepupal stage, in control medial niches, this fusion protein was detected in CCS, and sometimes in transition cells, but not in other TF cells (Fig 6A-6A", yellow bracket, green arrowhead, and green bracket, respectively). In striking contrast, upon depletion of Bab1 and Bab2 in hhG+ cells, dpp-nlsGFP was not 
expressed in the posterior-most $h h G+$ cells positive for $\mathrm{Tj}$ and located next to GCs in the medial region of the ovary, that we have characterized as CCs (Fig 6B-6B", yellow bracket).

Another $d p p$ transcriptional reporter, $d p p P 4-l a c Z$, contains a much smaller region of the cis-regulatory sequences present in dpp-nlsGFP. dppP4-lacZ was shown to be expressed in CCs as endogenous $d p p$, but also in TF cells in adult wild-type niches (Li et al., 2016), thereby not fully recapitulating the endogenous $d p p$ expression pattern. We found here that in the medial region of prepupal control ovaries, this reporter, like dpp-nlsGFP and endogenous dpp (Matsuoka et al., 2013), was expressed in CCs (Fig 6C-6C", yellow bracket) and often in transition cells (Fig 6C-6C', green arrowhead), but not in other TF cells (Fig 6C$6 C^{\prime \prime}$, green bracket). These $d p p P 4-l a c Z+C C s$ were in contact with pMad+/Vasa+ GSCs (Fig $6 C^{\prime}$, white arrowheads). In medial $h h G+$ cells depleted of Bab1 and Bab2, dppP4-lacZ was not or very faintly expressed (Fig 6D-6D") and this correlated with the absence of pMad in adjacent GCs (Fig 6D', white arrowheads). Altogether, these results indicate that Bab1 and Bab2 are necessary for $d p p$ transcription in CCs at the prepupal stage. In addition, since $h h G+$ cells depleted of Bab proteins express some CC markers but do not express dpp transcriptional reporters, they must thus be considered only as "CC-like".

\section{bab regulates Engrailed/Invected accumulation in TF cells, but not in CCs, during larval} stages

Like the bab genes, engrailed and its paralog invected (en/inv) have also been shown to be involved in proper TF formation (Bolívar et al., 2006). In addition, evidence strongly suggests that En directly controls $d p p$ expression in adult CCs (Luo et al., 2017). Therefore, we addressed whether bab function may act upstream of en/inv in larval stages, and thereby would impact $d p p$ transcriptional regulation indirectly through en/inv. In order to explore possible functional interactions between Bab and En/Inv proteins, we tested whether en/inv expression in niche cells of prepupal ovaries depends on Bab proteins. It has been reported that en/inv are specifically expressed in GSC niche cells as of the beginning of TF formation in the larval ovary (Allbee et al., 2018; Forbes et al., 1996; Green and Extavour, 2012; Mendes and Mirth, 2016). In addition, we found here a significant difference in En/Inv levels between TF cells and CCs in control prepupal niches (hs-FLP; FRT-bab ${ }^{A R O 7} / F R T-G F P$ and $h h G>G F P$, Fig $7 A$ and $7 A^{\prime}$ and Fig $7 B$ and $7 B^{\prime}$, green and yellow brackets, respectively), with a three-fold higher level in control TF cells than CCs (hs-FLP; GFP, Fig 7D). Induction of mitotic TF cell 
clones homozygous for $b a b^{A R O 7}$, a null allele for both bab1 and bab2 (Couderc et al., 2002), and marked by absence of GFP expression (Fig 7A, green arrowhead) showed that En/Inv levels were reduced by two-fold, cell autonomously in $b a b^{A R O 7}$ TF cell clones ( $h s-F L P ; b a b^{A R O 7}$ ) when compared to control TF cells (hs-FLP; GFP) in the same ovaries (Fig 7A and 7A', green arrowhead compared to green bracket, and Fig 7D for quantification). In contrast, En/Inv levels in $b a b^{A R O 7} \mathrm{CC}$ clones (Fig 7A and 7A', yellow arrowheads) were not significantly different from wild-type CCS in the same ovaries (Fig 7A and 7A', yellow arrowheads compared to yellow bracket, and Fig 7D for quantification).

Using RNAi to knockdown bab locus function in niche cells ( $h h G>G F P, b a b 1^{1 R}, b a b 2^{I R}$ ovaries), similar results were obtained, with En/Inv levels being significantly lower in anterior-most $h h G+$ cells depleted for Bab1 and Bab2 (Fig 7C', green arrowheads) than in control TFs (Fig 7B', green brackets), while present at the same levels in posterior-most $h h G+$ cells depleted for Bab1 and Bab2 in contact with GCs (Fig 7C', yellow arrowheads) than in control CCs (Fig 7B', yellow brackets, and Fig 7E for quantification). Taken together these results show that bab function is required cell-autonomously in TF cells of larval ovaries to ensure high En/Inv accumulation in these cells. On the contrary, bab function is not required in CCs to ensure normal level of En/Inv. Therefore, the function of Bab proteins for normal $d p p$ expression in CCs cannot be linked to their control of En/Inv accumulation in these cells.

\section{Depletion of Engrailed/Invected in niches does not prevent either TF formation or initial} GSC establishment

As shown above, strong depletion of Bab proteins in TF cells led to both lower accumulation of En/Inv in these cells and highly defective TF morphogenesis. Moreover, elimination of En/Inv by induction of mitotic TF cell clones homozygous for the $\mathrm{en}^{E} / \mathrm{inv}^{E}$ null mutant allele, affecting both paralogs, was previously shown to lead to a mild defect in alignment of TF cells in larval ovaries, which was aggravated by pupal stages (Bolívar et al., 2006). We thus tested whether depletion of En/Inv in all niche cells would phenocopy the $b a b$ loss of function strong TF morphogenesis defect. To achieve strong depletion of en/inv in niches cells, we used a bab-Gal4 driver (hereafter named babG), expressed in all ovarian somatic cells including niche cells (Cabrera et al., 2002) and Gal80 $80^{\text {TS }}$ which allows Gal4 activity at the Gal $80^{T S}$ restrictive temperature $\left(29^{\circ} \mathrm{C}\right)$ and impedes Gal4 activity at the Gal $80^{\mathrm{TS}}$ permissive temperature $\left(18^{\circ} \mathrm{C}\right)$. When the babG driver was expressed during all 
larval development, very strong En/Inv depletion was obtained in prepupal ovary niches (Fig $8 B$ and Fig 9B, $B^{\prime}$ ) compared to controls (Fig $8 A$ and Fig $9 A, A^{\prime}$ ). Under these conditions, normally formed TFs were present (Fig 8B-8B" compared to control in $8 \mathrm{~A}-8 \mathrm{~A}^{\prime \prime}$ ), and the number of TFs per ovary (Fig 8C), as well as the number of TF cells per TF (Fig 8D) and of CCs per TF (Fig 8E) were not significantly different from controls. These results indicate that en/inv function is not required for individual TF formation. Thus, even though bab function is required for accumulation of high levels of En/Inv in TF cells, the function of $b a b$ in TF formation most likely does not depend on en/inv function since these two genes are not required in this process.

In adult ovary GSC niches, en/inv is known to be necessary for GSC maintenance through its regulation of $d p p$ expression in CCs likely by direct binding of En to $d p p$ regulatory sequences (Luo et al., 2017; Rojas-Ríos et al., 2012). We tested whether en/inv gene functions are also necessary for GSC establishment in ovaries by the prepupal stage. Both control and En/Inv-depleted ovaries displayed niches with high and low pMad+ GSCS (Fig 9A and 9A' and Fig $9 \mathrm{~B}$ and 9B', red and pink arrowheads, respectively) and the proportion of TFs associated with GSCs was similarly high in both contexts $191.3 \%$ and 92.7\%, respectively, Fig 9C). The average number of GSCs per ovary, however, was about $30 \%$ lower in ovaries depleted of En/Inv when compared to control ovaries (Fig 9D). This difference corresponds to a lower proportion of high pMad+ GSCs per ovary in the en/inv RNAi context (about half as many, 29.6 vs. 13.7 on average, Fig 9D). Therefore, En/Invdepleted niches are almost all associated with GSCs but they contain fewer GSCs per niche than control niches and in particular fewer high-pMad+ GSCs per niche (Fig 9C). It is possible that En/Inv-depleted niches initially recruit GSCs with high pMad levels normally during niche formation, but that these niches do not have the capacity to maintain GSCs efficiently to the prepupal stage that was analyzed. In order to address this possibility, we tested the fate of GSCs, recruited initially within larval En/Inv-depleted niches, in young adult female siblings of the same genotype (Gal80 $\left.0^{T S}, b a b G>e n^{I R}, i n v^{I R}\right)$. GSC niches of these 1 day-old female siblings maintained at $29^{\circ} \mathrm{C}$ as of the prepupal stage (Fig 9E-9E'”') were efficiently depleted of En/Inv (Fig 9E-9E'"'), compared to niches from control females (Fig 9F-9F"') always maintained at $18^{\circ} \mathrm{C}$ (Fig 9F-9F"', yellow brackets indicate CCS). En/Inv depletion was associated with $100 \%$ of germaria lacking pMad+ GSCs (Fig 9E'", blue arrowheads compared to control in $9 \mathrm{~F}^{\prime \prime \prime}$, red and pink arrowheads, and Fig 9l), with a majority of germaria even 
devoid of any Vasa+ GCs (Fig 9H and 9l). This result indicates that all newly established GSCS in each niche of prepupal ovaries depleted of En/Inv (Fig 9B', red and pink arrowheads) were no longer present at adulthood (Fig 9E'”, blue arrowheads). This result supports the hypothesis that in the absence of En/Inv, GSCs are established at larval stages but that they are not maintained during pupal stages. This is supported by the fact that partial reexpression of en/inv in niche cells from the onset of pupariation $\left(29^{\circ} \mathrm{C}\right.$ to $18^{\circ} \mathrm{C}$ switch, Fig $9 \mathrm{G}$ 9G'", yellow bracket) substantially rescued the adult GSC loss phenotype (Fig 9G-9G'", pink arrowhead) from $0 \%$ to more than $60 \%$ (Fig 9l). Altogether, these results strongly suggest that en/inv functions are not essential for initial GSC establishment within newly formed niches during larval stages. However, they are essential for GSC maintenance in niches starting from at least the beginning of the pupal stage, but possibly even earlier, as soon as GSCs are established.

\section{Overexpression of bab2 in germaria leads to GSC-like tumors}

Since Bab proteins are necessary for TF morphogenesis and initial GSC establishment, we then asked whether these proteins could be sufficient to induce formation of ectopic TFs and/or GSCs. With this aim, we expressed UAS-bab1 or UAS-bab2 constructs with the C587Gal4 driver (called C587G hereafter) at either $25^{\circ} \mathrm{C}$ or $29^{\circ} \mathrm{C}$. C587G is expressed in most, but not all, somatic cells of developing female gonads (Zhu and Xie, 2003). With these tools, we generated prepupal ovaries with higher levels of Bab1 in ICs than in control ovaries (S2B Fig, arrowheads point to several ICs, compare to S2A Fig, bracket) and ectopic Bab1 in more basal somatic cells. For Bab2, higher levels were particularly obtained in ICs compared to the control (S2C' Fig, arrowheads point to ICs, compare to S2A', bracket). We found that ICs with higher Bab1 or Bab2 levels than normal did not exhibit En/Inv accumulation just as control ICs (S2B"and S2C" Fig, arrowheads compare to S2A", bracket). In addition, we did not detect any obvious change in Bab1 levels in cells overexpressing bab2, nor any change in Bab2 levels in cells ectopically expressing Bab1 (S2B' Fig compared to S2A' Fig and S2C Fig compared to S2A Fig). Finally, none of these conditions appeared to affect the organization of pupal ovaries (S2 Fig).

In adult germaria, C587G has been shown to be expressed only in ECs and early prefollicular cells (Song et al., 2004). In order to test the effect of expressing bab1 ectopically or over-expressing bab2 in adult ovaries, we analyzed ovaries from 10-day old females raised 
at $29^{\circ} \mathrm{C}$ as of eclosion. Under these conditions, we detected ectopic Bab1 in ECs and prefollicular cells near the middle of germaria (Fig 10B, $B^{\prime}$ compared to $10 \mathrm{~A}, \mathrm{~A}^{\prime}$, blue and yellow arrowheads, respectively). As for Bab2, significantly higher levels were induced in both ECs and prefollicular cells than in the control (Fig 10C-10C" compared to 10A-10A", blue and yellow arrowheads, respectively). The levels of Bab1 and Bab2 accumulation in ECs and prefollicular cells using UAS transgenes did not appear different from endogenous levels of these two proteins in niche cells of the same germaria (Fig 10B' and 10C', blue and yellow arrowheads point to ECs and prefollicular cells, respectively), compared to yellow brackets (CCs)). In ECs and prefollicular cells ectopically expressing bab1 or overexpressing bab2, no cross-regulation between the two genes was apparent (Fig 10B', $\mathrm{B}^{\prime \prime}$ and Fig 10C',C", yellow arrowheads). Moreover, abnormal accumulation of Bab1 and Bab2 in ECs and prefollicular cells did not lead to any obvious morphological changes of these cells.

However, with respect to GCs (Vasa+), in ovaries overexpressing bab2, all germaria contained more than the normal number of GSCs as marked by the presence of pMad, some in continuity with the niche and others at ectopic locations (Fig 10F and 10F' and Fig 10G, pink brackets and pink arrowheads, respectively, compared to the control in Fig 10D and 10D'). Among these germaria, 75\% exhibited more than $20 \mathrm{GSCs}$ and 25\% between 5 and 20 GSCs ( $n=137)$, compared to 0 and $10 \%(n=87)$, respectively, for control germaria. In many cases, overexpression of bab2 led to the formation of huge germaria with GSC-like tumors as marked by the presence of spectrosomes (Hts+ cytoplasmic structures, Fig 10H). In these tumorous germaria, no ectopic En/Inv was detected in somatic cells outside of the endogenous niche (Fig 10G', yellow dotted line and yellow bracket, respectively). Upon ectopic expression of bab1, only 4 germaria out of 133 exhibited ectopic GCs in which the BMP pathway was activated (Fig $10 \mathrm{E}$ and $10 \mathrm{E}^{\prime}$, pink arrowheads). Altogether, these results show that bab2 overexpression in somatic cells outside of the adult GSC niche is sufficient to induce activation of the BMP pathway in adjacent GCs, thereby producing numerous ectopic GSCs and tumorous germaria. Since bab1 ectopic expression did not affect GSC number significantly, either Bab1 does not have the equivalent capacity as Bab2, or is not expressed at the correct level in the condition tested to provoke an effect. In addition, since somatic Bab2 overexpression does not produce ectopic En/Inv expression, we conclude that Bab2 GSC-like tumor induction, associated with BMP pathway activation in GCs, does not require en/inv function in bab2 overexpressing cells. 


\section{DISCUSSION}

\section{Bab 1 and Bab2 are redundant in precursor niche cells for functional GSC niche formation}

The two bab paralogs encode transcription factors that have been shown to be required for leg proximal-distal differentiation, ovary morphogenesis and sexually-dimorphic abdomen pigmentation (Bartoletti et al., 2012; Cabrera et al., 2002; Godt and Laski, 1995; Kopp et al., 2000; Sahut-Barnola et al., 1995). bab1 and bab2 display partially overlapping expression patterns in these three organs (leg, ovary, abdomen) (Couderc et al., 2002; Kopp et al., 2000; Williams et al., 2008). Indeed, Bab1 is always present with Bab2, while Bab2 is present alone in additional territories in the leg and ovary (Couderc et al., 2002). The question of redundancy in function between Bab paralogs has been hampered in part by their overlapping expression (at least during leg and ovary development), but especially by the lack of any known bab2 molecularly null mutation not affecting bab1. Indeed, for bab1, the $b a b^{A 128}$ allele has been shown to be associated with complete loss of Bab1 without affecting Bab2 levels (confirmed in the present study), but no equivalent allele has been characterized for bab2 (Chalvet et al., 2011; Couderc et al., 2002). Two studies have now addressed the question of redundancy between the two bab genes using tissue-specific expression of bab1 and bab2 RNAi constructs, for abdomen pigmentation (Roeske et al., 2018) and the present study for ovarian GSC niche formation. Roeske et al. (Roeske et al., 2018) showed that both $b a b 1$ and bab2 are necessary for repression of pigmentation of female abdomen segments $A 5$ and A6. However, since depletion of both proteins leads to a much stronger female pigmentation defect than depletion of each one separately, this and other results led the authors to propose that 4 doses of Bab proteins, provided in the wildtype by the presence of the two paralogs, are needed to repress expression of a pigmentation gene, yellow, in the female abdomen. In addition, their work and that of Couderc et al. (Couderc et al., 2002) indicate equivalent capacities for Bab1 and Bab2 since ectopic expression of either one at the same level in males leads to similar abnormal pigmentation of $A 5$ and $A 6$. Taken together, these results suggest redundancy between Bab1 and Bab2 for sexually-dimorphic abdominal pigmentation.

In developing ovary niches, our results indicate full redundancy between bab1 and bab2 for TF formation and strongly suggest that this is also the case for GSC establishment. 
Therefore, in ovarian GSC niches, two doses of Bab proteins seem sufficient for these processes. In contrast, ectopic and overexpression of bab1 and bab2, respectively, outside of the GSC niche, did not produce similar effects. Indeed, somatic overexpression of bab2 in germaria led to production of GSC-like tumors, while under similar conditions bab1 did not significantly affect germaria. This may not be so surprising since in other contexts Bab proetin activities do not seem fully equivalent. For example, bab mutant rescue experiments conducted with respect to leg and ovary developmental mutant phenotypes have shown that both Bab1 and Bab2 are able to rescue the mutant phenotypes, but that Bab2 does so more efficiently (Bardot et al., 2002). In addition, bab2 has a much larger expression domain than bab1 in the ovary, and removing bab2 from both GSC niches and the rest of the somatic cells has a much stronger effect on ovary morphogenesis than removing bab2 only from niche cells (Green and Extavour, 2012). Altogether, bab1 and bab2 thus seem to have redundant functions in the tissues where they are co-expressed and bab2 has additional functions in tissues where bab1 is not expressed.

\section{A newly-identified role for Bab proteins in GSC establishment through dpp expression in CCs}

The BMP family member Dpp, known to be emitted principally by CCs within niches germaria, has been shown to be essential for maintenance of GSC status in the adult (Luo et al., 2017; Song et al., 2004; Xie and Spradling, 1998). During larval to pupal transition, Dpp produced by niches has also been shown to be required for GSC establishment and proliferation (Gilboa and Lehmann, 2004; Matsuoka et al., 2013; Zhu and Xie, 2003). When both bab1 and bab2 functions were removed from precursor niche cells, these cells were not organized into TFs at the pupal stage and GSC establishment, as evidenced by activated Dpp signal transduction, was largely absent. Absence of GSCs does not seem to be due to a defect in CC specification, since the expression pattern of several markers, the combination of which distinguishes CCs, allowed us to identify cells presenting CC characteristics among bab deficient niche cells correctly positioned adjacent to GCs. Indeed, like wild-type CCs in prepupal ovaries, these cells produce $\mathrm{Tj}$ and En/Inv, express P1444Z-lacZ (albeit at somewhat lower levels than in control cells), are devoid of Delta at the plasma membrane but present rare Delta-containing vesicles and are activated for the Notch signaling pathway reporter $E(S p l) m \beta$-CD2. Importantly, $\mathrm{Tj}$ and the Notch pathway have been shown to be 
necessary for CC specification (Hsu and Drummond-Barbosa, 2011; Panchal et al., 2017; Song et al., 2007; Yatsenko and Shcherbata, 2018). Therefore, several aspects of CC specification do not depend on bab gene functions.

However, in bab deficient niche cells expressing CC markers, the expression of two $d p p$ transcriptional reporters, normally observed in control CCs, was absent. Therefore, Bab proteins act as activators of $d p p$ transcription in CCs, directly or indirectly. Bab proteins are thus necessary for at least one very important property of CCs, that of expressing high levels of $d p p$, which is essential for their function as GSC niche cells (Liu et al., 2010, 2015; Luo et al., 2017; Wang et al., 2008; Xie and Spradling, 1998). Hence, bab requirement in GSC establishment likely relies on its control of $d p p$ expression. Thus, our results reveal an as yet unknown function for Bab proteins in niches for GSC establishment.

TF cell specification seems more perturbed than that of CCs in Bab1 and Bab2depleted niche cells. Though $\mathrm{Tj}$ expression was absent as in control TF cells, En/Inv levels were two-fold lower, and P1444-lacZ expression and membranous Delta were undetectable, unlike in control TF cells. It is therefore not possible to attribute a clear identity to these cells. Incorrect TF cell specification could explain why these cells are unable to undergo proper TF morphogenesis. Taken together these results indicate that in developing niches Bab proteins are necessary for TF cell specification and for the functionality of CCs for GSC establishment.

\section{En/Inv are not essential for TF formation nor GSC establishment, but seem necessary for GSC maintenance as of niche formation}

In the ovary, en/inv are expressed specifically in niches during their formation. Our results show that their functions, however, are not necessary for niche formation. Indeed, we found that when En/Inv were depleted in developing niches by RNAi, the same number of correctly formed TFs, TF cells and CCs were found as in controls. This is consistent with the observation of normal larval ovaries upon En/Inv depletion in Allbee et al. (Allbee et al., 2018), using a different niche cell Gal4 driver and the same en/inv RNAi transgenes as used in our study. In contrast, another study, based on induction of clones of ovarian somatic cells homozygous for a null allele for both en/inv, concluded that these genes are involved specifically for proper TF cell alignment within individual stacks in larval ovaries (Bolívar et 
al., 2006). The difference with the results presented here, may be related to the different approaches used to abolish en/inv expression. With the RNAi approach used here, all niche cells are depleted of En/Inv, while en/inv null mutant clone induction used in Bolívar et al. (Bolívar et al., 2006) led to the formation of stacks with both wild-type and mutant TF cells. Possible heterogeneity in the identity between the two populations of cells may have impeded normal interaction between the cells during the stacking of TF cells.

Our results also indicate that En/Inv are not essential for initial GSC establishment since when they were strongly reduced, the proportion of prepupal niches associated with at least one GSC was the same as in the control. However, if left to develop until eclosion, these ovaries had lost all their GSCs suggesting that GSCs could not be maintained without the presence of En/Inv in niche cells. When expression of en/inv was restored in niche cells from the beginning of pupariation, then GSCs were present in adult germaria. These results suggest that the known function of En/Inv for GSC maintenance in the adult (Luo et al., 2017; Rojas-Ríos et al., 2012), probably through direct regulation of $d p p$ expression (Luo et al., 2017), may be additionally necessary for this process earlier, as of niche formation during larval/prepupal stages. Indeed, at the prepupal stages the overall number of GSCs upon En/Inv depletion was already about $30 \%$ less than that in the control, and these GSCS showed a globally lower level of BMP signal activation than that in the control. We propose that Bab proteins are essential for initial GSC establishment through control of $d p p$ expression in CCS during larval stages, and that as of this point, En/Inv are required for GSC maintenance, likely also through $d p p$ regulation in these same cells. Therefore, taken together, our results suggest that the molecular players responsible for regulation of $d p p$ expression in CCs as of niche formation, first for GSC establishment and, subsequently, for GSC maintenance may not be the same. Consistent with this, the absence of Bab proteins in CCs does not affect En/Inv levels in these same cells, and GSC establishment is nonetheless highly affected. However, it is not possible to completely exclude that En/Inv participate to $d p p$ expression and GSC establishment and/or proliferation, in parallel to Bab, but to a lesser extent than Bab. Indeed, in prepupal ovaries, the region depleted of both Bab proteins is associated with the presence of almost no GSCs, while upon En/Inv depletion at this same stage, about two thirds of the normal number of GSCs are present.

A newly-identified gene necessary for ovary morphogenesis is $L m \times 1 a$, encoding a LIM-homeodomain transcription factor expressed in somatic apical cells, TF cells and CCs in 
the prepupal ovary (Allbee et al., 2018). Transcriptomic analysis revealed that en/inv are positively regulated by $L m \times 1 a$ in ovaries at the larval to pupal transition, but $L m \times 1 a$ was shown to be necessary for proper organization and differentiation of niche cells, unlike En/Inv. Indeed, Lmx1a niche mutant phenotypes resemble those of bab genes and strong genetic interactions were demonstrated between $L m \times 1 a$ and $b a b$ genes. In addition, Bab proteins were shown to be necessary for $L m \times 1 a$ expression and thus Lmx1a is a potential Bab target, direct or indirect, to be tested in the future for mediation of the role of Bab in niches for their correct formation.

\section{bab2 overexpression leads to production of GSC-like ovarian tumors in adults}

We have shown that in developing niches Bab proteins are necessary for BMP pathway activation and GSC establishment. We therefore also addressed whether ectopic Bab proteins in somatic cells outside of the niche could be sufficient to induce BMP pathway activation in adjacent GCs. During ovary development, we did not detect a significant effect upon bab1 ectopic expression or bab2 overexpression in non-niche somatic cells. In contrast, in adult germaria, overexpression of bab2 in ECs and prefollicular cells led to the formation of GSC-like germarial tumors indicating that Bab2 is sufficient for acquisition of GSC status. The GSC-like germarial tumor phenotype was similar to that previously described for germarial overexpression of $d p p$ (Song et al., 2004; Xie and Spradling, 1998) or en (Eliazer et al., 2014; Luo et al., 2017). Importantly, the phenotype observed in germaria overexpressing bab2 was not a consequence of ectopic expression of En/Inv, since in these germaria, En/Inv were found only in endogenous niches as in the control, far from ectopic GSCs. Thus, ectopic Dpp activity in GCs induced by bab2 overexpression is independent of en/inv function. Therefore, our results indicate that Bab controls Dpp pathway activation both in GSCs of endogenous niches and in ectopic GSCs in adult germaria, and that, in both cases, this is independent of En/Inv. Since we show here that bab is necessary for $d p p$ expression in CCS and for GSC recruitment during niche formation, it is possible that the presence of ectopic GSCs in adult germaria overexpressing bab2 somatically could be due to ectopic activation of $d p p$ expression in these cells. Interestingly, inhibition of the JAK/STAT pathway in niche cells has also been shown to result in reduction in GSC numbers associated with low $d p p$ expression in niches, without affecting expression of other CC markers (Decotto and Spradling, 2005; Lopez-Onieva et al., 2008; Wang et al., 2008). In addition, ectopic activation 
of the JAK/STAT pathway in the germarium is sufficient to induce ectopic GSC-like cells associated with ectopic $d p p$ expression but not with ectopic CC specification. Bab interaction with this signaling pathway remains to be explored.

\section{MATERIAL AND METHODS}

\section{Fly stocks}

We used hedgehog-Gal4 (gift from P. Therond and hereafter called $h h G$ ), a Gal4-expressing enhancer trap insertion in the $h h$ locus, to target niche cells during their differentiation, and $b a b^{P G a / 4-2}$ (gift from J.L. Couderc and hereafter called babG), a Gal4-expressing enhancer trap insertion in the bab locus, to drive expression in all somatic cells of the larval ovary (Cabrera et al., 2002) and present study). These drivers were combined with UAS-dicer2 and tubGal80 ${ }^{T S}$ (gift from J. Montagne) when indicated. Two different UAS-GFP transgenes (one associated with a Nuclear Localization Signal sequence, Bloomington Drosophila Stock Center, BDSC 4476, and one without, gift from A. Boivin), were used in combination with the $h h G$ driver to mark niche cells. The RNAi lines UAS-bab1 $1^{I R}$ (Vienna Drosophila Stock Center, VDRC 50285) and UAS-bab2 $2^{I R}$ (VDRC 49042), and the bab1 null allele bab ${ }^{A 128}$ (Godt et al., 1993) (gift from D.Godt) were used for the bab loss-of-function analysis. $b a b^{A R 07}, F R T 80 B / T M 6 B$ (gift from M. Boube) and hs-FLP;; ubi-GFP, FRT80B (gift from J. Montagne) lines were used to generate $b a b$ null mitotic cell clones. $b a b^{A R O 7}$ is a deletion inactivating both bab1 and bab2 (Couderc et al., 2002). UAS-en ${ }^{I R}$ (VDRC 35697) and UAS-inv ${ }^{I R}$ (BDSC 41675) were used for the engrailed/invected knockdown analysis. The enhancer reporter line $P\{P Z\} 1444$ (P1444-lacZ) (Margolis and Spradling, 1995) and bamP-GFP, a GFP transcriptional reporter for bam expression ((Chen and McKearin, 2003a), Kyoto DGRC 118177) were used as cell-type specific markers. $E(s p l) m \beta-C D 2$, in which the sequence encoding rat CD2 protein is inserted downstream of the Enhancer of Split [E(spl)m $\beta$ ] promoter that is activated by Notch signaling, was used as a readout of Notch pathway activity (de Celis et al., 1998) (gift from A. Bardin). dpp-GFP (VDRC 318464) and dpp-nlsGFP (VDRC 318414) (Sarov et al., 2016) and P4-lacZ (Li et al., 2016) (gift from R. Xi) were used as $d p p$ transcriptional reporters. $d p p-n / s G F P$ contains $43766 \mathrm{bp}$ of the $d p p$ genomic locus. P4lacZ contains $1494 \mathrm{bp}$ of a dpp enhancer region. To drive ectopic and over-expression of 
bab1 and bab2, we used C587-Gal4 (Zhu and Xie, 2003) (gift from T. Xie), UAS-bab1 (BDSC, 6939) and UAS-bab2 $2^{4-66}$ (Bardot et al., 2002; Couderc et al., 2002) (gift from A. Kopp).

\section{Experimental conditions}

For all the experiments, flies were raised on standard cornmeal food.

For pupal analysis of the effect of bab and engrailed/invected (en/inv) depletion in niche cells with $b a b^{A 128}, h h G, b a b G$, and RNAi lines, crosses were started at $25^{\circ} \mathrm{C}$, parents removed 24-to-48h later, descendants were then transferred to $29^{\circ} \mathrm{C}$ and ovaries from 1-to-6 hour old prepupae (white pupae) were dissected.

To generate $b a b$ mosaic mutant prepupal ovaries, $b a b^{A R 07}, F R T 80 B / T M 6 B$ flies were crossed to $h s-F L P ; ; ~ u b i-G F P, F R T 80 B$ flies at $25^{\circ} \mathrm{C}$. Clones marked by absence of GFP were induced by one 1-hour heat shock at $38^{\circ} \mathrm{C}$ at the end of the second instar larval stage, and prepupae were dissected for ovary analysis $50 \mathrm{~h}$ after heat shock.

Transient expression of the UAS-engrailed $d^{/ R}$ and UAS-invected $d^{/ R}$ transgenes was achieved using Tub Gal80 ${ }^{T S}$ and babG. For analysis of adult (1-day old) ovaries upon RNAi-mediated knockdown of engrailed/invected (en/inv), two conditions were used: $18^{\circ} \mathrm{C}$ (from crosses to dissection) for the control and $29^{\circ} \mathrm{C}$ (crosses at $25^{\circ} \mathrm{C}$ and transferred 24 -to-48h later to $29^{\circ} \mathrm{C}$ ) for the depletion of En/Inv. To test the effect of the depletion only during niche formation, en/inv were reexpressed at the prepupal stage by shifting prepupae from $29^{\circ} \mathrm{C}$ to $18^{\circ} \mathrm{C}$ for the rest of development.

To analyze the effect, at prepupal stage, of bab1 ectopic expression and bab2 overexpression in somatic cells of the ovaries during larval stages, crosses were conducted at $25^{\circ} \mathrm{C}$ for 24 -to- $48 \mathrm{~h}$ and descendants were then transferred either to $29^{\circ} \mathrm{C}$ or maintained at $25^{\circ} \mathrm{C}$ until dissection.

For bab1 and bab2 gain-of-function experiments in the adult, individuals were raised at $18^{\circ} \mathrm{C}$ and then shifted to $29^{\circ} \mathrm{C}$ after eclosion. Ovaries from 10 day-old females were dissected.

\section{Immunostaining}

Prepupal ovaries were dissected in PBS medium, fixed in 4\% formaldehyde (R1026, Agar Scientific) for 20 to 30 minutes, and washed $3 \times 10$ minutes in PBT (PBS supplemented with 0.3\% tritonX-100 - Sigma T8787). Ovaries were then incubated in a blocking solution (PBTA: PBT supplemented with $1 \%$ BSA - Sigma A3059) for a minimum of 20 minutes. Primary 
antibodies were diluted in PBTA and ovaries were incubated in this solution for 6 hours at room temperature or overnight at $4^{\circ} \mathrm{C}$. The following primary antibodies were used: rabbit anti-Smad3 (1:200, ab52903, Abcam); rabbit anti-Bab1 (1:4000, gift from T. Williams); rabbit anti-GFP (1:200, FP-37151B, Interchim); rat anti-Bab2 (1:4000, gift from J-L. Couderc); rat IgM anti-Vasa (1:500, Developmental Studies Hybridoma Bank-DSHB); mouse anti-En/Inv recognizing both paralogs (1:200, 4D9, DSHB); guinea-pig anti GFP (1:200, 132 002, Synaptic System); mouse anti- $\beta-G a l$ (1:400, 40-1a, DSHB,); guinea pig anti-Traffic Jam (1:5000, gift from D. Godt); mouse anti-Delta (1:200, C594.9B, DSHB), mouse anti-rat CD2 (1:100, MCA154GA, Biorad); mouse anti hts (1:200, DSHB). After primary antibody incubation, tissues were washed in PBT $3 \times 10 \mathrm{~min}$, and incubated in PBTA for at least 30 minutes before incubation in secondary antibodies in PBTA. The following secondary antibodies were used: donkey anti-mouse Cy3 (715-165-151- Jackson Laboratories); and the following ones from Thermo Fisher Scientific: chicken anti-rabbit 488 (A21441); goat anti-rabbit 568 (A11011); goat anti-guinea pig 488 (A11073), anti-mouse 488 (A11029); anti-rat IgM 647 (A21248); anti-rat IgG 647 (A21247). Nuclei were detected with DAPI (1:200, 1mg/ml) and F-actin with Phalloidin:647 (1:200, 65906, Sigma). After secondary antibody incubation, tissues were washed 3x10 min in PBT, and mounted between slide and coverslip in DABCO (D27602, Sigma) with $70 \%$ glycerol. For larval ovaries, a spacer was positioned between the slide and the coverslip to avoid ovary squashing. The lateral side of the prepupal ovary was identified by its contact with the fat body.

\section{Fluorescence microscopy, image analysis and statistics}

Images were acquired with a $40 x$ or $63 x$ objective on a confocal laser-scanning microscope Leica TCS SP8 equipped with $405 \mathrm{~nm}, 488 \mathrm{~nm}, 552 \mathrm{~nm}$, and $638 \mathrm{~nm}$ emission diodes. Same settings were used between the different genotypes in each experiment. Prepupal ovaries were acquired with $1 \mu \mathrm{m}$ steps between each section and AOTF/EOF compensation was used to increase diode percentage during acquisition using the LasX software. Adult ovary images were acquired with 0.5 or $1 \mu \mathrm{m}$ steps between each section.

Images represent either a projection of 4 consecutive sections or a 3D reconstruction of the entire tissue. Images were processed with Fiji (Schindelin et al., 2012).

Counting TF number in prepupal ovaries was achieved through immunodetection of niche cell markers, such as GFP ( $h h G+$ cells), Bab1 or En/Inv, or DAPI labeling of flattened TF cell 
nuclei. Both lateral views and $3 D$ reconstructions from the anterior pole of ovaries were used for this quantification. To distinguish TF cells and CCs among niche cells, labeling of Factin which delimits cell contours and/or DAPI labeling of flattened TF cell nuclei was used. Niche cells with contours covering the entire width of the TF and with flattened nuclei were considered as TF cells, and more basal cells contacting GCs that did not cover the width of the TF and had round nuclei, as CCs (See Fig1B, compare green and yellow brackets, respectively).

$h h G+$ cell flattening of control prepupal ovaries ( $h h G>G F P$ ) or upon depletion of Bab proteins $\left(h h G>G F P, b a b 1^{l R}, b a b 2^{l R}\right)$ was quantified with GFP immunostaining marking $h h G+$ cells and fluorescent phalloïdin-bound F-actin marking cell contours in projections of 5 consecutive sections separated by $1 \mu \mathrm{m}$. The width and height between cell contours of individual hhG+ cells were measured independently, and the degree of flattening corresponded to the ratio between the width and height.

For comparison of En/Inv levels between control niche cells and niche cells depleted of Bab proteins ( $b a b^{A R O 7}$ null allele and RNAi-mediated depletion), the confocal section presenting the biggest area for individual nuclei was used to quantify fluorescence intensity of En/Inv levels using the RawIntDen tool in Fiji.

To quantify GSC number in both prepupal ovaries and adult niches, we used nuclear pMad staining in GCs as a readout of Dpp pathway activity. pMad staining levels in nuclei were very variable between GCs of same ovary and between ovaries of a same experiment ranging from very high to very low when detected with Royal LUT. GCs presenting low but unambiguous nuclear pMad staining were considered separately from GCs with high pMad+ GCs. GCs with high or low pMad levels were both considered as GSCs since pMad detection reflects a high or moderate activity of Dpp/BMP pathway (see Figure 1C-C').

Prism 7 (GraphPad Software) was used for statistical tests and for the generation of graphs. Values are presented as means + s.e.m., p-value calculated using a two-tailed Student's ttest when two conditions were compared or an ANOVA one-way test for comparisons between multiple conditions. Results are indicated with: NS (Not Significant - p>0.05); * $(0.05>p>0.01) ; * *(0.01>p>0.001) ; * * *(0.001>p>0.0001)$ and $* * * *(p<0.0001)$. 


\section{REFERENCES}

Aguado, B.A., Bushnell, G.G., Rao, S.S., Jeruss, J.S., and Shea, L.D. (2017). Engineering the premetastatic niche. Nat. Biomed. Eng. 1.

Allbee, A.W., Rincon-Limas, D.E., and Biteau, B. (2018). Lmx1a is required for the development of the ovarian stem cell niche in Drosophila. Development 145, dev163394.

Bardot, O., Godt, D., Laski, F.A., and Couderc, J.-L. (2002). Expressing UAS-bab1 and UAS-bab2: A comparative study of gain-of-function effects and the potential to rescue the bric à brac mutant phenotype. Genesis 34, 66-70.

Bartoletti, M., Rubin, T., Chalvet, F., Netter, S., Dos Santos, N., Poisot, E., Paces-Fessy, M., Cumenal, D., Peronnet, F., Pret, A.-M., et al. (2012). Genetic Basis for Developmental Homeostasis of Germline Stem Cell Niche Number: A Network of Tramtrack-Group Nuclear BTB Factors. PLoS ONE 7.

Bolívar, J., Pearson, J., López-Onieva, L., and González-Reyes, A. (2006). Genetic dissection of a stem cell niche: The case of the Drosophila ovary. Dev. Dyn. 235, 2969-2979.

Cabrera, G.R., Godt, D., Fang, P.-Y., Couderc, J.-L., and Laski, F.A. (2002). Expression pattern of Gal4 enhancer trap insertions into the bric à brac locus generated by $\mathrm{P}$ element replacement. Genesis 34, 62-65.

de Celis, J.F., Tyler, D.M., de Celis, J., and Bray, S.J. (1998). Notch signalling mediates segmentation of the Drosophila leg. 10.

Chaharbakhshi, E., and Jemc, J.C. (2016). Broad-complex, tramtrack, and bric-à-brac (BTB) proteins: Critical regulators of development. Genesis 54, 505-518.

Chalvet, F., Bartoletti, M., and Théodore, L. (2011). Ovary phenotype and expression of bab1 and bab2paralogs in the ovary of two mutants of bab locus in Drosophila melanogaster. Drosoph Info Serv 158-162.

Chen, D., and McKearin (2003a). A discrete transcriptional silencer in the bam gene determines asymmetric division of the Drosophila germline stem cell. Development 130, 1159-1170.

Chen, D., and McKearin, D. (2003b). Dpp Signaling Silences bam Transcription Directly to Establish Asymmetric Divisions of Germline Stem Cells. Curr. Biol. 13, 1786-1791.

Cohen, E.D., Mariol, M.-C., Wallace, R.M.H., Weyers, J., Kamberov, Y.G., Pradel, J., and Wilder, E.L. (2002). DWnt4 Regulates Cell Movement and Focal Adhesion Kinase during Drosophila Ovarian Morphogenesis. Dev. Cell 2, 437-448.

Couderc, J.-L., Godt, D., Zollman, S., Chen, J., Li, M., Tiong, S., Cramton, S.E., Sahut-Barnola, I., and Laski, F.A. (2002). The bric à brac locus. 15.

Decotto, E., and Spradling, A.C. (2005). The Drosophila ovarian and testis stem cell niches: similar somatic stem cells and signals. Dev. Cell 9, 501-510.

Eliazer, S., Palacios, V., Wang, Z., Kollipara, R.K., Kittler, R., and Buszczak, M. (2014). Lsd1 restricts the number of germline stem cells by regulating multiple targets in escort cells. PLoS Genet. 10, e1004200.

Ermolaeva, M., Neri, F., Ori, A., and Rudolph, K.L. (2018). Cellular and epigenetic drivers of stem cell ageing. Nat. Rev. Mol. Cell Biol. 19, 594.

Forbes, A.J., Spradling, A.C., Ingham, P.W., and Lin, H. (1996). The role of segment polarity genes during early oogenesis in Drosophila. 12.

Gancz, D., Lengil, T., and Gilboa, L. (2011). Coordinated Regulation of Niche and Stem Cell Precursors by Hormonal Signaling. PLoS Biol. 9.

Gilboa, L. (2015). Organizing stem cell units in the Drosophila ovary. Curr. Opin. Genet. Dev. 32, 3136.

Gilboa, L., and Lehmann, R. (2004). Repression of Primordial Germ Cell Differentiation Parallels Germ Line Stem Cell Maintenance. Curr. Biol. 14, 981-986.

Godt, D., and Laski, F.A. (1995). Mechanisms of cell rearrangement and cell recruitment in Drosophila ovary morphogenesis and the requirement of bric à brac. 15.

Godt, D., Couderc, J.-L., Cramton, S.E., and Laski, F.A. (1993). Pattern formation in the limbs of 
Drosophila: bric à brac is expressed in both a gradient and a wave-like pattern and is required for specification and proper segmentation of the tarsus. 14.

Green, D.A., and Extavour, C.G. (2012). Convergent evolution of a reproductive trait through distinct developmental mechanisms in Drosophila. Dev. Biol. 372, 120-130.

Green, D.A., Sarikaya, D.P., and Extavour, C.G. (2011). Counting in oogenesis. Cell Tissue Res. 344, 207-212.

Greenspan, L.J., de Cuevas, M., and Matunis, E. (2015). Genetics of Gonadal Stem Cell Renewal. Annu. Rev. Cell Dev. Biol. 31, 291-315.

Gustavson, E., Goldsborough, A.S., Ali, Z., and Kornberg, T.B. (1996). The Drosophila engrailed and invected genes: partners in regulation, expression and function. Genetics 142, 893-906.

Hsu, H.-J., and Drummond-Barbosa, D. (2011). Insulin signals control the competence of the Drosophila female germline stem cell niche to respond to Notch ligands. Dev. Biol. 350, 290300.

Kai, T., and Spradling, A. (2004). Differentiating germ cells can revert into functional stem cells in Drosophila melanogaster ovaries. Nature 428, 564-569.

Kaplan, R.N., Riba, R.D., Zacharoulis, S., Bramley, A.H., Vincent, L., Costa, C., MacDonald, D.D., Jin, D.K., Shido, K., Kerns, S.A., et al. (2005). VEGFR1-positive haematopoietic bone marrow progenitors initiate the pre-metastatic niche. Nature 438, 820-827.

Kopp, A., Duncan, I., and Carroll, S.B. (2000). Genetic control and evolution of sexually dimorphic characters in Drosophila. Nature 408, 553-559.

Lai, C.-M., Lin, K.-Y., Kao, S.-H., Chen, Y.-N., Huang, F., and Hsu, H.-J. (2017). Hedgehog signaling establishes precursors for germline stem cell niches by regulating cell adhesion. J. Cell Biol. 216, 1439-1453.

Li, M.A., Alls, J.D., Avancini, R.M., Koo, K., and Godt, D. (2003). The large Maf factor Traffic Jam controls gonad morphogenesis in Drosophila. Nat. Cell Biol. 5, 994-1000.

Li, X., Yang, F., Chen, H., Deng, B., Li, X., and Xi, R. (2016). Control of germline stem cell differentiation by Polycomb and Trithorax group genes in the niche microenvironment. Dev. Camb. Engl. 143, 3449-3458.

Liu, M., Lim, T.M., and Cai, Y. (2010). The Drosophila Female Germline Stem Cell Lineage Acts to Spatially Restrict DPP Function Within the Niche. Sci. Signal. 3, ra57-ra57.

Liu, Z., Zhong, G., Chai, P.C., Luo, L., Liu, S., Yang, Y., Baeg, G.-H., and Cai, Y. (2015). Coordinated niche-associated signals promote germline homeostasis in the Drosophila ovary. J. Cell Biol. 211, 469-484.

Lopez-Onieva, L., Fernandez-Minan, A., and Gonzalez-Reyes, A. (2008). Jak/Stat signalling in niche support cells regulates dpp transcription to control germline stem cell maintenance in the Drosophila ovary. Development 135, 533-540.

Lu, T., Wang, S., Gao, Y., Mao, Y., Yang, Z., Liu, L., Song, X., Ni, J., and Xie, T. (2015). COP9-Hedgehog axis regulates the function of the germline stem cell progeny differentiation niche in the Drosophila ovary. Development 142, 4242-4252.

Luo, L., Siah, C.K., and Cai, Y. (2017). Engrailed acts with Nejire to control decapentaplegic expression in the Drosophila ovarian stem cell niche. Development 144, 3224-3231.

Margolis, J., and Spradling, A. (1995). Identification and behavior of epithelial stem cells in the Drosophila ovary. Dev. Camb. Engl. 121, 3797-3807.

Matsuoka, S., Hiromi, Y., and Asaoka, M. (2013). Egfr signaling controls the size of the stem cell precursor pool in the Drosophila ovary. Mech. Dev. 130, 241-253.

McKearin, D., and Ohlstein, B. (1995). A role for the Drosophila bag-of-marbles protein in the differentiation of cystoblasts from germline stem cells. Development 121, 2937-2947.

Mendes, C.C., and Mirth, C.K. (2016). Stage-Specific Plasticity in Ovary Size Is Regulated by Insulin/Insulin-Like Growth Factor and Ecdysone Signaling in Drosophila. Genetics 202, 703719.

Panchal, T., Chen, X., Alchits, E., Oh, Y., Poon, J., Kouptsova, J., Laski, F.A., and Godt, D. (2017). Specification and spatial arrangement of cells in the germline stem cell niche of the Drosophila 
ovary depend on the Maf transcription factor Traffic jam. PLoS Genet. 13.

Plaks, V., Kong, N., and Werb, Z. (2015). The Cancer Stem Cell Niche: How Essential Is the Niche in Regulating Stemness of Tumor Cells? Cell Stem Cell 16, 225-238.

Prager, B.C., Xie, Q., Bao, S., and Rich, J.N. (2019). Cancer Stem Cells: The Architects of the Tumor Ecosystem. Cell Stem Cell 24, 41-53.

Roeske, M.J., Camino, E.M., Grover, S., Rebeiz, M., and Williams, T.M. (2018). Cis-regulatory evolution integrated the Bric-à-brac transcription factors into a novel fruit fly gene regulatory network. ELife 7.

Rojas-Ríos, P., Guerrero, I., and González-Reyes, A. (2012). Cytoneme-Mediated Delivery of Hedgehog Regulates the Expression of Bone Morphogenetic Proteins to Maintain Germline Stem Cells in Drosophila. PLoS Biol. 10.

Sahut-Barnola, Dastugue, Bernard, and Couderc, J.-L. (1996). (PDF) Terminal filament cell organization in the larval ovary of Drosophila melanogaster: Ultrastructural observations and pattern of divisions.

Sahut-Barnola, I., Godt, D., Laski, F.A., and Couderc, J.-L. (1995). Drosophila Ovary Morphogenesis: Analysis of Terminal Filament Formation and Identification of a Gene Required for This Process. Dev. Biol. 170, 127-135.

Sarikaya, D.P., and Extavour, C.G. (2015). The Hippo Pathway Regulates Homeostatic Growth of Stem Cell Niche Precursors in the Drosophila Ovary. PLoS Genet. 11.

Sarikaya, D.P., Belay, A.A., Ahuja, A., Dorta, A., Green, D.A., and Extavour, C.G. (2012). The roles of cell size and cell number in determining ovariole number in Drosophila. Dev. Biol. 363, 279289.

Sarov, M., Barz, C., Jambor, H., Hein, M.Y., Schmied, C., Suchold, D., Stender, B., Janosch, S., KJ, V.V., Krishnan, R., et al. (2016). A genome-wide resource for the analysis of protein localisation in Drosophila. ELife 5, e12068.

Sato, T., Ogata, J., and Niki, Y. (2010). BMP and Hh Signaling Affects Primordial Germ Cell Division in Drosophila. Zoolog. Sci. 27, 804-810.

Schindelin, J., Arganda-Carreras, I., Frise, E., Kaynig, V., Longair, M., Pietzsch, T., Preibisch, S., Rueden, C., Saalfeld, S., Schmid, B., et al. (2012). Fiji: an open-source platform for biological-image analysis. Nat. Methods 9, 676-682.

Song, X., zhu, and Xie (2002). Germline Stem Cells Anchored by Adherens Junctions in the Drosophila Ovary Niches. Science 296, 1855-1857.

Song, X., wong, and kawase (2004). Bmp signals from niche cells directly repress transcription of a differentiation-promoting gene, bag of marbles, in germline stem cells in the Drosophila ovary. Development 131, 1353-1364.

Song, X., Call, G.B., Kirilly, D., and Xie, T. (2007). Notch signaling controls germline stem cell niche formation in the Drosophila ovary. Development 134, 1071-1080.

Tseng, C.-Y., Su, Y.-H., Yang, S.-M., Lin, K.-Y., Lai, C.-M., Rastegari, E., Amartuvshin, O., Cho, Y., Cai, Y., and Hsu, H.-J. (2018). Smad-Independent BMP Signaling in Somatic Cells Limits the Size of the Germline Stem Cell Pool. Stem Cell Rep. 11, 811-827.

Wang, X., and Page-McCaw, A. (2018). Wnt6 maintains anterior escort cells as an integral component of the germline stem cell niche. Dev. Camb. Engl. 145.

Wang, L., Li, Z., and Cai, Y. (2008). The JAK/STAT pathway positively regulates DPP signaling in the Drosophila germline stem cell niche. J. Cell Biol. 180, 721-728.

Ward, E.J., Shcherbata, H.R., Reynolds, S.H., Fischer, K.A., Hatfield, S.D., and Ruohola-Baker, H. (2006). Stem Cells Signal to the Niche through the Notch Pathway in the Drosophila Ovary. Curr. Biol. 16, 2352-2358.

Williams, T.M., Selegue, J.E., Werner, T., Gompel, N., Kopp, A., and Carroll, S.B. (2008). The Regulation and Evolution of a Genetic Switch Controlling Sexually Dimorphic Traits in Drosophila. Cell 134, 610-623.

Xie, T., and Spradling, A.C. (1998). decapentaplegic Is Essential for the Maintenance and Division of Germline Stem Cells in the Drosophila Ovary. Cell 94, 251-260. 
Xie, T., and Spradling, A.C. (2000). A Niche Maintaining Germ Line Stem Cells in the Drosophila Ovary. Science 290, 328-330.

Yatsenko, A.S., and Shcherbata, H.R. (2018). Stereotypical architecture of the stem cell niche is spatiotemporally established by miR-125-dependent coordination of Notch and steroid signaling. Development 145, dev159178.

Zhao, dong, and li (2018). Targeting cancer stem cells and their niche_perspectives for future therapeutic targets and strategies | Elsevier Enhanced Reader.

Zhu, C.-H., and Xie, T. (2003). Clonal expansion of ovarian germline stem cells during niche formation in Drosophila. Development 130, 2579-2588. 


\section{Fig 1. Specific cell types of the developing Drosophila ovary.}

(A) Schematic drawings of a developing ovary from mid L3 to prepupal stages and of an adult germarium, with all cell types indicated and represented by a different color. Anterior is to the top and for the developing ovary, medial is to the left. TF cells: Terminal Filament cells. TC: Transition Cell. CCs: Cap cells. ISCs: Inner Sheath Cells. GSCs: Germline Stem Cells. ICs: Intermingled Cells. ECs: Escort cells. The round spherical structure in germ cells during larval and prepupal stages represent the spectrosome and the red branched structures in germ cell cysts in the germarium represent fusomes, which are derived from spectrosomes. Spectrosomes and fusomes are cytoplasmic, cytoskeletal structures, and are used as specific germ cell markers.

(B-D') Whole mount immunostaining of wild type prepupal ovaries (projections of adjacent confocal sections). Anterior is up, medial is to the left. Scale bar: $10 \mu \mathrm{m}$. ( $\left.B^{\prime}, C^{\prime \prime}\right)$ Higher magnification of the niche region of the corresponding ovary. (B, $\left.B^{\prime}\right)$ Engrailed/Invected (En/Inv) (red) mark the niche cell nuclei specifically and F-actin labeling (grey) marks all cell membranes. TF cells (green bracket) are characterized by a flat shape, with flat nuclei accumulating high levels of En/Inv, while CCs (yellow bracket) are cuboidal, with weaker En/Inv expression level. The triangular TC (green arrowhead) is located between the TF cells and the CCs. (C) En/Inv (green) marks the nuclei of niche cells, and Vasa (blue) marks the cytoplasm of Germ Cells (GCs). ( $\left.C^{\prime}, C^{\prime \prime}\right)$ In the most anterior GCs, in contact with niche cells, the BMP signaling pathway is activated as evidenced by accumulation of pMad (red) at high (red arrowheads) or low (pink arrowheads) levels. GCs accumulating pMad and in contact with CCs are considered as GSCs. CCs (yellow arrowheads) and other peripheral posterior cells (blue arrowhead) accumulating pMad (red) can be differentiated from GCs since they do not accumulate Vasa (blue). The most posterior GCs, engaged into differentiation, do not accumulate pMad (white arrowheads). (D) Bab2 accumulates in the nuclei of all somatic cells but at a higher level in niche TF cells and CCS (green and yellow brackets, respectively, compared to blue bracket). (D') Bab1 accumulates in niche TF cells and CCs and is also reported here for the first time to be present at low levels in ICs at this stage (blue bracket). 
Fig. 2. Depletion of Bab1 and Bab2 together in niches of larval ovaries impedes Terminal Filament formation.

(A-E) Whole mount immunostaining of prepupal ovaries (projections of adjacent confocal sections) for detection of GFP (green), Bab1 (pink) and Bab2 (yellow). Nuclei are labeled with DAPI (grey). Anterior is up, medial is left. Scale bars: $10 \mu \mathrm{m}$. $\left(A^{\prime}, B^{\prime}, C^{\prime}, D^{\prime}, E^{\prime}\right)$ Higher magnification of the niche region of the corresponding ovaries. $\left(A^{\prime \prime}, B^{\prime \prime}, C^{\prime \prime}, D^{\prime \prime}, E^{\prime \prime}\right)$ Anterior view of ovaries using 3D reconstruction. Each GFP (green) circle corresponds to one Terminal Filament (TF) cross section. (A) Control ovary expressing a UAS-GFP construct under the control of a hedgehog(hh)-Gal4 driver ( $h h G>G F P$ ). This driver is expressed as a decreasing medial (yellow dotted lines) to lateral (blue dotted lines) gradient in the TFs and CCs of niches as evidenced by the GFP (green) reporter. Moreover, in a single niche, the expression pattern of $h h G$ is mosaic between TF cells and is always low in CCs at the base of each TF. Bab2 (yellow) accumulates in nuclei of all somatic ovarian cells, but at a higher level in TF cell and CC nuclei. Bab1 (magenta) accumulates at a high level in TF cell and CC nuclei. (B) Prepupal ovary expressing RNAi against bab2 (hhG>GFP, bab2 $\left.{ }^{1 R}\right)$. Consistent with the expression profile of the $h h G$ driver, Bab2 depletion is obtained in all medial TF cells but not in all CCS at the base of these TFs (yellow dotted lines), and is not obtained in lateral $h h G+$ cells (blue dotted lines). Bab1 (magenta) accumulation is not noticeably affected. (C) Prepupal ovary mutant for bab1 $\left(b a b^{A 128}, h h G>G F P\right)$. Bab1 (magenta) is not detectable in medial or lateral niches, and the level of Bab2 (yellow) is not noticeably affected. For both genetic contexts $(B, C)$, the morphology of the TFs and TF cells appear unaffected compared to the control (A). (D) Prepupal ovary expressing RNAi against bab2 coupled with a homozygous bab1 null mutation $\left(b a b^{A 128} ; h h G>G F P, b a b 2^{1 R}\right)$. As in $\mathrm{B}$, Bab2 (yellow) depletion is obtained in medial (yellow dotted line) but not lateral $h h G+$ cells (blue dotted line) and Bab1 (magenta) is absent. (E) Prepupal ovary expressing RNAi against bab1 and bab2 $\left(h h G>G F P, b a b 1^{1 R}, b a b 2^{1 R}\right)$. Consistent with B, Bab1 (magenta) and Bab2 (yellow) depletion is obtained in medial (yellow dotted line) but not lateral $h h G+$ cells (blue dotted line). For both genetic contexts $(D, E)$, in the medial part of the ovary (yellow dotted lines), the $h h G+$ cells fail to flatten, stack and form TFs. (F-I) Graphs comparing different parameters related to TF formation, in control ovaries ( $h$ hG>GFP) and in the ovaries depleted of Bab1 and/or Bab2. (F) The number of TFs per ovary is not significantly different between the control and the ovaries depleted of Bab1 or Bab2. However, the ovaries depleted of both Bab1 and Bab2 have significantly fewer TFs per ovary than the control. (G) The number of TF cells (TFCS) per TF is not different between control ovaries and ovaries depleted of Bab1 or Bab2. (H) Control hhG+ cells are significantly flatter than those knocked down for bab1 and bab2, with flattening measured as the ratio of the width and height.

(I) The total number of $h h G+$ cells is comparable in control ovaries and ovaries depleted of Bab1 and Bab2 $\left(b a b^{A 128} ; h h G>G F P, b a b 2^{l R}\right)$. Values are presented as means + s.e.m. $p$-values are calculated 
bioRxiv preprint doi: https://doi.org/10.1101/689323; this version posted July 3, 2019. The copyright holder for this preprint (which was not certified by peer review) is the author/funder. All rights reserved. No reuse allowed without permission.

using a one-way ANOVA test for $\mathrm{F}$ and $\mathrm{G}$ and a two-tailed t-test for $\mathrm{H}$ and I. $\mathrm{n}$ : sample size; NS: Not Significant $(p>0.05) ; * * * *:(p<0.0001)$. 


\section{Fig 3. Bab1 and Bab2 depletion in developing niches impedes Germline Stem Cell establishment.}

(A-E) Whole mount immunostaining of prepupal ovaries (projections of adjacent confocal sections). Nuclei are labelled with DAPI (grey). Anterior is up, medial is left. Scale bars: $10 \mu \mathrm{m}$. The yellow dotted lines encircle the medial Terminal Filament (TF)/region, and the blue dotted lines, the lateral TF of each ovary. The transgenes and mutations present are indicated to the left of each row. $\left(A^{\prime}, B^{\prime}, C^{\prime}, C^{\prime \prime}, D^{\prime}, E^{\prime}\right)$ Higher magnification of the niche region (GFP, green) of the corresponding ovary. $\left(A, A^{\prime}\right)$ In a control ovary, pMad (red) immunostaining is detected in the anterior-most Germ Cells (GCs), marked with the presence of Vasa protein (blue). These Vasa+/pMad+ are in direct contact with the niches and are considered as Germline Stem Cells (GSCS). (B,B') Ovary mutant for bab1 $\left(b a b^{A 128} ; h h G>G F P\right)$. No significant difference was observed when compared to the control, regarding the presence of Vasa+/pMad+ GSCs in contact with the niches. $\left(A^{\prime}, B^{\prime}\right)$ The $C C$ s and some peripheral posterior cells (arrowheads) accumulate pMad (red), but can be differentiated from GCs since they do not accumulate Vasa (blue). (C-C") Ovary expressing UAS-nISGFP, UAS-bab2 ${ }^{R}$ and UAS-dicer2 under control of hedgehog(hh)-Gal4 (hhG>GFP, dicer2, $b a b 2^{I R}$ ). In both the medial part of the ovary, where depletion of Bab2 protein (grey) is strong in CCs ( $C^{\prime \prime}$, yellow dotted lines), and in lateral niches where it is not ( $C^{\prime \prime}$, blue dotted lines), GSCs, marked by nuclear pMad staining (red), are associated with CCs. (D-E) Ovaries depleted of Bab1 and Bab2 using two genetic contexts, bab ${ }^{A 128}$; hhG $>G F P$, $b a b 2^{I R}(D)$ and $h h G>G F P, b a b 2^{I R}, b a b 2^{I R}(E)$. In the medial part of the ovary where we have shown that Bab protein depletion is strong, $h h G+$ cells do not form TFs (yellow dotted lines), Vasa+ GCs (blue) are present, but almost none of them are GSCs since they are not pMad+. However, in the lateral region, where we have shown Bab protein depletion is weak, normal TFs are formed (blue dotted lines) and these are associated with Vasa+/pMad+ GSCs. (F) Graph comparing the overall number of GSCs per ovary in control ovaries and in ovaries depleted of Bab1 alone $\left(b a b^{A 128}\right)$ or both Bab1 and Bab2 using the two different genetic contexts. A significantly lower number of GSCs per ovary were obtained only when Bab1 and Bab2 were depleted together compared to the control, with no significant difference between the two mutant contexts. Moreover, the majority of GSCs in both mutant contexts were found in the lateral part of the ovary were TFs from (blue bar) and very few in the medial part (about 3 GSC per ovary, yellow bar). Values are presented as means +s.e.m. p-values are calculated using a one-way ANOVA test. $\mathrm{n}$ : sample size; NS: Not Significant $(\mathrm{p}>0.05)$; ****: $(p<0.0001)$. 
Fig 4. Some Germ Cells in contact with niche cells depleted of Bab proteins begin to differentiate into cystoblasts.

(A-B') Whole mount immunostaining of prepupal ovaries (projections of adjacent confocal sections) for detection of $\beta$-Galactosidase ( $\beta$ - Gal, Royal Lookup Table (LUT) Fiji, indicating signal intensity, inset to the right), GFP (green) and Vasa (red). Nuclei are labeled with DAPI (grey). Anterior is up, medial is left. Scale bars: $10 \mu \mathrm{m}$. $h h G+$ cells revealed by the detection of $\beta$-Gal (Royal LUT) are encircled in yellow and blue in the medial and lateral regions of the ovaries, respectively. $\left(A^{\prime}, B^{\prime}\right)$ Higher magnification of the niche region of the corresponding ovaries in (A,B). Germ Cells (GCs) are marked with Vasa (red). (A') In a control ovary (bamP-GFP, hhG>lacZ), the differentiating GCs expressing the GFP bam transcriptional reporter (green) are mainly found one-cell diameter away from the $\beta-G a l+(h h G+)$ cells (yellow and blue dotted lines). (B') In ovaries depleted of Bab1 and Bab2, the differentiating GCs expressing the GFP bam transcriptional reporter (green) can also be observed in direct contact with $\beta-G a l+(h h G+)$ cells (white arrowhead). (C) Quantification of the number of GFP+ differentiating GCs in contact with $\beta-G a l+(h h G+)$ cells. In the ovaries depleted of Bab1 and Bab2, significantly more GFP+ differentiating GCs are found in contact with $\beta-G a l+(h h G+)$ cells and most of these cells are found in the medial part of the ovary where depletion of the Bab proteins is strong (yellow bar) rather than laterally where it is low (blue bar). Values are presented as means +s.e.m. $p$-values are calculated using a two-tailed t-test. $n$ : sample size; $* * * *:(p<0.0001)$. 
Fig 5. bab1 and bab2 functions in niche cells are not necessary for expression of several CC specification markers.

(A-F) Whole mount immunostaining of the medial region of prepupal ovaries (projections of adjacent confocal sections). Nuclei are labelled with DAPI (grey). Anterior is up. Scale bars: $10 \mu \mathrm{m}$. (A-A'"',C$\mathrm{C}^{\prime \prime}, \mathrm{E}-\mathrm{E}^{\prime \prime}$ ) correspond to the control ovaries. One hhG>GFP niche is encircled in each panel (pink dotted line). (B-B"',D-D",F-F") correspond to bab1 and bab2 RNAi-mediated knockdown ovaries. The entire cluster of $h h G+$ cells depleted of Bab1 and Bab2 is encircled in each panel (pink dotted line). $(A, C, E)$ The green and yellow brackets indicate Terminal Filament (TF) cells and Cap Cells (CCS), respectively, and the green arrowheads point to transition cells. (B,D,E) The green and yellow brackets indicate the anterior- and posterior-most $h h G+$ cells, respectively. (A-A'") In the control ovaries, the two CCs markers, nuclear P1444-lacZ (Royal LUT) and nuclear Traffic Jam (red), are detected in CCs (yellow bracket) and in the transition cell (green arrowhead). Nuclear P1444-lacZ is also detected at a weaker level in some TF cells. The Germ Cells (GCs), marked with Vasa protein (red and cytoplasmic), in direct contact with niche cells (white arrowheads) show nuclear pMad (green) and are thus considered as Germinal Stem Cells (GSCs). (B-B'") Posterior-most medial hhG+ cells depleted of Bab1 and Bab2, express both CC markers ( $\mathrm{Tj}$ and P1444-lacZ) and are adjacent to GCs which do not present pMad+ ( $\mathrm{B}^{\prime \prime \prime}$, arrowheads) and thus are not considered to be GSCs. (C-C") In control ovaries, Delta (Royal LUT) accumulates at the plasma membrane and in cytoplasmic vesicles in TF cells, is detected in vesicle around the transition cell (green arrowhead) and sometimes in CCS (arrow). (D-D") Upon depletion of Bab1 and Bab2 in $h h G+$ cells, Delta is not present at the plasma membrane, but is found in some vesicles (arrows). (E-E",F-F") In both the control (E-E") and upon depletion of Bab1 and Bab2 in hhG+ cells (F-F"), the Notch pathway transcriptional reporter $E(s p l) m b-C D 2$ is expressed since CD2 (Royal LUT) accumulates at the plasma membrane of TF cells / anterior-most $h h G+$ cells (green brackets) and of CCs / posterior-most $h h G+$ cells (yellow brackets), also accumulating $\mathrm{Tj}$. 


\section{Fig 6. bab1 and bab2 functions in niche cells are required for $d p p$ expression.}

(A-D) Whole mount immunostaining of the medial region of prepupal ovaries (projections of adjacent confocal sections). Nuclei are labeled with DAPI (grey). Anterior is up. Scale bars: $10 \mu \mathrm{m}$. (A'-D') GCs are visualized with cytoplasmic Vasa (red). One hhG>GFP niche is encircled in images of control ovaries ( $A-A ", C-C ")$ and green and yellow brackets indicate Terminal Filament (TF) cells and Cap Cells (CCs), respectively. The green arrowheads point to transition cells. (B-B", D-D") The entire cluster of medial $h h G+$ cells is encircled in images of bab1 and bab2 knockdown ovaries, and green and yellow brackets indicate the anterior- and posterior-most $h h G+$ cells, respectively. $(A, B)$ Somatic cells are marked with Bab2 (green). (A-A") In a control ovary (dpp-nlsGFP, hhG>+), dpp-nlsGFP (Royal LUT) is specifically expressed in the Cap Cells (CCs) (yellow bracket) along with nuclear Traffic Jam (Tj, red) and sometimes in the transition cell (green arrowhead), but not in Terminal Filaments (green

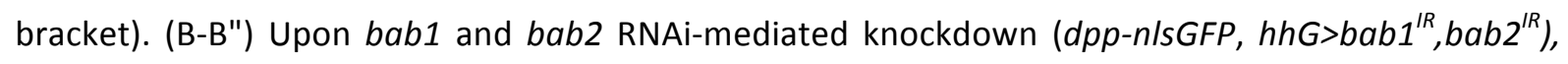
$d p p$-nlsGFP is not expressed in posterior-most $h h G+$ cells (yellow brackets) that are positive for the nuclear CC marker Tj (red), and in contact with the Germ Cells (GCs) marked with cytoplasmic Vasa (red). (C-C") In a control ovary (dpp-P4lacZ, hhG>GFP), dpp-P4lacZ expression is found in CCs (yellow brackets) in contact with GSCs marked with cytoplasmic Vasa (red) and nuclear pMad+ (green) (white arrowheads) and often in the transition cell (green arrowhead). (D-D") Upon bab1 and bab2 RNAimediated knockdown ( $\left.d p p-P 4 l a c Z, h h G>b a b 1^{I R}, b a b 2^{I R}\right), d p p-P 4 l a c Z$ expression is not detected in the posterior-most $h h G+$ cells, positive for the CC marker Tj (yellow brackets), which correlates with an absence of pMad in the Vasa+ underlying GCs (white arrowheads). 
Fig 7. bab locus regulates Engrailed/Invected accumulation in Terminal Filament cells but not in

\section{Cap Cells.}

(A-C') Whole mount immunostaining of prepupal ovaries (projections of adjacent confocal sections) for detection of GFP (green) and En/Inv shown in Royal LUT ( $\left.A^{\prime}\right)$ and red ( $B^{\prime}$ and $C^{\prime}$ ). F-actin labeling is shown in red $(A)$ and grey $\left(B-C^{\prime}\right)$. Anterior is up, medial is left. Scale bars: $10 \mu \mathrm{m} .\left(A, A^{\prime}, B^{\prime}\right)$ Green and yellow brackets indicate Terminal Filament (TF) cells and Cap Cells (CCs), respectively. (C,C') The entire cluster of medial $h h G+$ cells depleted for Bab1 and Bab2 is encircled, and green and yellow arrowheads indicate the anterior- and posterior-most $h h G+$ cells, respectively. $\left(A, A^{\prime}\right)$ Niche region of a mosaic ovary ( $h s-F L P$; FRT-bab $b^{A R 07} / F R T$-GFP), containing mitotic cell clones homozygous for the $b a b^{A R O 7}$ mutation and marked by absence of GFP (green and yellow arrowheads). In $b a b^{A R 07}$ mutant Terminal Filament (TF) cells (green arrowheads), the signal for Engrailed/Invected (En/Inv) is lower than that in wild type TF cells (green brackets). However, in bab ${ }^{A R O 7}$ mutant Cap Cells (CCs) (yellow arrowheads), the level of En/Inv is similar to its endogenous level in wild type CCs (yellow bracket). $(\mathrm{B}, \mathrm{C})$ Prepupal ovaries expressing GFP in the niche cells ( $h h G>G F P$ ), as well as RNAi against bab1 and bab2 (hhG>GFP, bab1 $1^{I R}, b a b 2^{I R)}$ in $\left.C\right)$. $\left(B^{\prime}, C^{\prime}\right)$ Higher magnification of the regions framed with dotted lines in the corresponding ovaries. The knockdown of bab1 and bab2 in TF cells and CCs leads to the presence of anterior-most cells $h h G+$ cells ( $C^{\prime}$, green arrowheads) with lower levels of En/Inv compared to TF cells in the control (B', green brackets). In contrast, the posterior-most hhG+ cells depleted of Bab1 and Bab2 ( $C^{\prime}$, yellow arrowheads), show a similar level of En/Inv compared to that in control CCs (B', yellow brackets). (D) Graph comparing the fluorescence intensity of En/Inv in control TF cells and CCs compared to the corresponding bab ${ }^{A R O 7}$ mitotic clonal cells (A). In $b a b^{A R O 7}$ mutant TF cells ( $h s-F L P, b a b^{A R 07}$ ), the En/Inv fluorescence intensity is 2-fold lower than in adjacent control TF cells ( $h s-F L P, G F P)$. However, in $b a b^{A R O 7}$ mutant CCs, the En/Inv fluorescence intensity is similar to that in the adjacent control CCs. (E) Graph comparing the fluorescence intensity of En/Inv in CCs in the control ( $\mathrm{B}^{\prime}$, yellow brackets) and in posterior-most $h h G+$ cells ( $\mathrm{C}^{\prime}$, yellow arrowheads) upon bab1 and bab2 RNAi-mediated knockdown. There is no significant difference between En/Inv levels in the two genetic contexts. Values are presented as means +s.e.m. p-values are calculated using a two-tailed t-test. $n$ : sample size; NS: Not Significant ( $>>0.05) ; * * * *: p<0.0001)$. 


\section{Fig 8. TF formation does not rely on en/inv function.}

(A-B) Whole mount immunostaining of prepupal ovaries (projections of adjacent confocal sections) for detection of Engrailed/Invected (En/Inv) (Royal LUT). Nuclei are labeled with DAPI (grey). F-actin labeling is shown in magenta and red ( $A, B$ and $A ", B "$, respectively). Anterior is up, medial is left. Scale bars: $10 \mu \mathrm{m}$. $\left(A^{\prime}, A^{\prime \prime}, B^{\prime}, B^{\prime \prime}\right)$ Higher magnifications of the niche regions of the corresponding ovaries in (A,B). (A-A") In a control ovary $\left(G a / 80^{T S}\right.$, babG>+) En/Inv accumulate in the niche cells: at high levels in Terminal Filament cells (TF cells; green bracket) and at lower levels in Cap Cells (CCs; yellow bracket). (B-B") In an ovary carrying transgenes targeting en/inv for RNAi (babG/UAS-en ${ }^{\prime R}$, $U A S-i n v^{I R}$ ) these two proteins are efficiently depleted (B), while TFs are well formed (B") and TF cells properly aligned within TFs ( $\left.\mathrm{B}^{\prime}\right)$. (C-E) Graphs comparing different parameters related to TF formation, between control ovaries and ovaries depleted of En/Inv. (C) number of TFs per ovary, (D) number of TF cells per TF and (E) number of CCs per TF. The TF cells (green brackets in A-A") were distinguished from CCs (yellow brackets in A-A") by their flattened nuclei and their flat shape, as determined by DAPI (grey, $A^{\prime}$ and $B^{\prime}$ ) and F-actin labeling (red, $A^{\prime \prime}$ and $B^{\prime \prime}$ ), respectively. CCs were distinguished from Intermingled Cells (ICS) using Bab1 immunostaining, which is high in CCs and low in ICs (data not shown) and from TF cells due to the round shape of their nuclei. None of these parameters were significantly different between control and En/Inv depleted ovaries. Values are presented as means +s.e.m. $p$-values are calculated using a two-tailed t-test. $n$ : sample size; NS: Not Significant $(p>0.05)$. 


\section{Fig 9. En/Inv are not necessary for GSC establishment but are involved in their maintenance.}

(A-B) Whole mount immunostaining of prepupal ovaries (projections of adjacent confocal sections) for detection of Engrailed/Invected (En/Inv) (green) and Vasa (Royal LUT). Nuclei are labeled with DAPI (grey). Anterior is up, medial is left. Scale bars: $10 \mu \mathrm{m}$. $\left(A^{\prime}, B^{\prime}\right)$ Higher magnifications of the niche regions of the corresponding ovaries in (A,B). In control ovaries (Gal80 $\left.{ }^{T S} ; b a b G>+\right)$ and ovaries depleted of En/Inv (Gal80 ${ }^{T S}$; babG/UAS-en ${ }^{I R}$, UAS-inv ${ }^{I R}$ ), Germinal Stem Cells (GSCs) with both high and low pMad levels are present (red and pink arrowheads, respectively). (C) Pie chart comparing the proportion of prepupal niches associated with: at least one GSC with a high level of pMad (red), at least one GSC with a low level of pMad (pink), GCs without nuclear pMad (blue) and no Germinal Cells (GC) (green). The depletion of En/Inv does not change the proportion of Terminal Filaments (TFs) associated with at least one GSC (sum of pink and red portions). (D) Graph comparing the number of GSCs per prepupal ovary in control and En/Inv depleted ovaries. The number of GSCs per ovary is significantly lower in En/Inv depleted ovaries (black bar), due to the significantly lower number of high-level pMad+ GSCs (red bar) and not to the number of low-level pMad+ GSCs (pink bar) which are not significantly different. Values are presented as means +s.e.m. p-values are calculated using a two-tailed t-test. $n$ : sample size; NS: Not Significant $(p>0.05) ; * * * *:(p<0.0001)$. (EH) Germaria from 1 day-old adult females carrying transgenes for temperature-controlled RNAi of en/inv (Gal80 ${ }^{T S}$; babG/UAS-en ${ }^{I R}$, UAS-inv ${ }^{I R}$ ), immunostained for En/Inv (green), pMad and Bab1 (Royal LUT) and Vasa (red). Nuclei are labelled with DAPI. Images are projection of confocal sections. Anterior is up and the scale bars represent $10 \mu \mathrm{m}$. The transgenes present and temperatures used for raising the flies are indicated on top of the images. $\left(E^{\prime}-E^{\prime \prime \prime}, F^{\prime}-F^{\prime \prime \prime}, G^{\prime}-G^{\prime \prime \prime}\right)$ Higher magnifications of the corresponding niche regions in $(E, F, G)$. (E-E"') Germarium of a female raised at $29^{\circ} \mathrm{C}$ from the $\mathrm{L} 1$ stage to dissection shows efficient depletion of En/Inv in Cap Cells (CCs) (9E", yellow bracket). This germaria does not contain any pMad+ GSCs (E'", blue arrowheads). (F-F"') Germarium of a female raised at $18^{\circ} \mathrm{C}$ from the $\mathrm{L} 1$ stage to dissection is not depleted of En/Inv in CCs ( $F^{\prime \prime}$, yellow bracket) and exhibits GSCs with high and low levels of pMad (F'", red and pink arrowheads, respectively). (GG"') Germarium of a female raised at $29^{\circ} \mathrm{C}$ from L1 stage and transferred to $18^{\circ} \mathrm{C}$ at the prepupal stage, showing the presence of En/Inv in CCs (G', yellow bracket) and a pMad+ GSCs (G'", pink arrowhead). (H) Germarium of a female raised at $29^{\circ} \mathrm{C}$ from the $\mathrm{L} 1$ stage to dissection showing efficient depletion of En/Inv in CCs (yellow bracket). This germaria does not contain any GCs (Vasa, red) close to the niche. (I) Pie chart comparing the proportion of germaria with: at least one GSC with a high level of pMad (red), at least one GSC with a low level of pMad (pink), GCs without nuclear pMad (blue), or no GCs (green). The re-expression of en/inv in ovaries from the prepupal stage onwards $\left(29->18^{\circ} \mathrm{C}\right.$ ) leads to partial rescue (from $0 \%$ to $62.6 \%$ ) of the proportion of germaria containing GSCs. 


\section{Fig 10. The overexpression of bab2 is sufficient to induce GSC expansion in adult germaria.}

(A-H) Whole mount immunostaining of prepupal ovaries germaria from 10-day old females (projections of adjacent confocal sections). DAPI nuclear labeling Anterior is up. Scale bars: $10 \mu \mathrm{m}$. (A$A^{\prime \prime}, D-D^{\prime}$ ) In a control (C587G>+), Bab1 (green, $A^{\prime}$ ) and Bab2 (red, $A^{\prime \prime}$ ) are present in niche cells, mainly in the Cap Cells (CCs) (yellow bracket) and overlying Terminal Filaments (TFs). Bab2 can also be detected faintly in some Escort Cells (ECS) (blue arrowhead, $\mathrm{A}^{\prime \prime}$ ) and more posterior somatic cells (yellow arrowhead, A"). (D,D') Three Germinal Stem Cells (GSCs) are marked with pMad (green) at high or low level (red and pink arrowhead, respectively). pMad also accumulates faintly in the maturating germline cyst (red bracket). (B-F') Ectopic expression of bab1 (C587G>UAS-bab1 - B', green) or overexpression of bab2 (C587G>UAS-bab1 - C", red) causes elevated accumulation of the corresponding proteins in ECs and prefollicular cells compared to the control ( $B^{\prime}, C^{\prime \prime}$ compare to $A^{\prime}, A^{\prime \prime}$, blue and yellow arrowheads, respectively). Cross regulation between bab1 and bab2 does not occur ( $\mathrm{B}^{\prime \prime}$ and $\mathrm{C}^{\prime}$, blue and yellow arrowheads). (E, $\left.\mathrm{E}^{\prime}\right)$ In rare cases, the ectopic expression of bab1 leads to the presence of ectopic pMad+ Germ Cells (GCs) (pink arrowhead). pMad is also detected faintly in the maturating germline cyst as in the control (red bracket). (F-G) Inducing higher levels of Bab2 in ECs and prefollicular cells leads to an increase in the number of pMad+ GSCs in continuity with the niche (pink bracket) or at ectopic locations in germaria (pink arrowheads). (G,G') Engrailed/ Invected are not detected in somatic cells (yellow dotted lines) in contact with the ectopic GSCs (pink arrowheads) compared to accumulation of these proteins in the endogenous niche (yellow bracket). (H) The overexpression of bab2 leads to the formation of huge germaria with GSC-like tumors marked by the presence of spectrosome-containing (Hts, yellow) GCs (Vasa, red). 
S1 Fig. Expression pattern of $d p p-G F P$ and $d p p$-nIsGFP transcriptional reporters in adult germaria. Whole mount immunostaining of adult germaria (projections of adjacent confocal sections) from females raised at $25^{\circ} \mathrm{C}$ for detection of GFP (green), Engrailed/Invected (En/Inv, red) and Traffic Jam/Vasa (Tj/Vasa, magenta). Nuclei are labeled with DAPI (grey). Anterior is up. Scale bars: $10 \mu \mathrm{m}$. $(\mathrm{A}, \mathrm{B})$ Germaria expressing dpp-GFP and dpp-nlsGFP, respectively. ( $\left.\mathrm{A}^{\prime}-\mathrm{A}^{\prime \prime \prime}, \mathrm{B}^{\prime}-\mathrm{B}^{\prime \prime \prime}\right)$ Higher magnification of the regions framed with dotted lines in the corresponding germaria. For both constructions, GFP (green) is present in the Cap Cells (yellow brackets) co-marked by nuclear Engrailed/Invected (En/Inv, red) and nuclear Traffic Jam (TJ, magenta), and in some Escort Cells (blue brackets), but absent from Terminal Filament cells (green brackets) marked by En/Inv (red). Expression of both GFP reporters was also detected in prefollicular cells (orange brackets). 
S2 Fig. The ectopic expression of bab1 or the overexpression of bab2 does not affect ovary morphogenesis.

(A-C) Whole mount immunostaining of prepupal ovaries (projections of adjacent confocal sections) from females raised at $25^{\circ} \mathrm{C}$. Anterior is up and medial is left. Scale bars: $10 \mu \mathrm{m}$. Nuclei are labeled with DAPI (grey). (A-A") Control ovary (C587-Gal4 (C587G)>+) showing the expression pattern of bab1 (strong expression in niche cells and weak in Intermingled Cells (ICs, white brackets). (B-B") The C587G driver coupled with UAS-bab1 allows the ectopic expression of bab1 in some ICS (arrowheads). (C-C') The C587G driver coupled with UAS-bab2 allows an overexpression of bab2 in some ICs (arrowheads). ( $\left.B^{\prime \prime}, C^{\prime \prime}\right)$ Neither of these constructs causes ectopic accumulation of En/Inv (red) in ICs overexpressing bab1 or bab2 (arrowheads). The overall organization of these ovaries does not seem disturbed compared to the control. 
bioRxiv preprint doi: https://doi.org/10.1101/689323; this version posted July 3, 2019. The copyright holder for this preprint (which was not certified by peer review) is the author/funder. All rights reserved. No reuse allowed without permission.

A
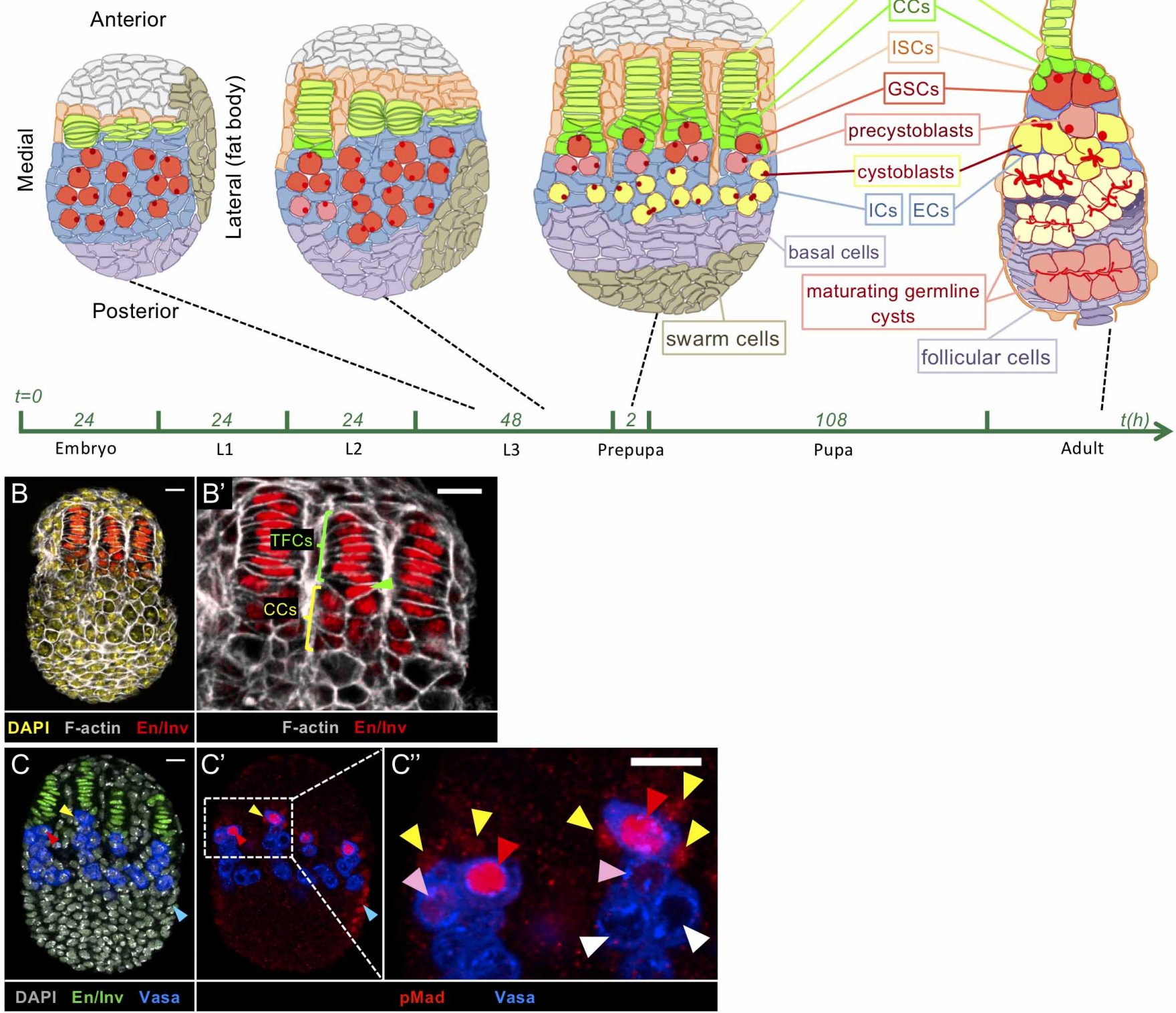

DAPI En/lnv Vasa
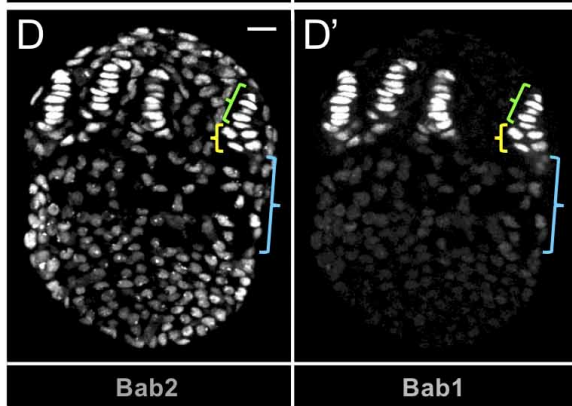

Fig 1 

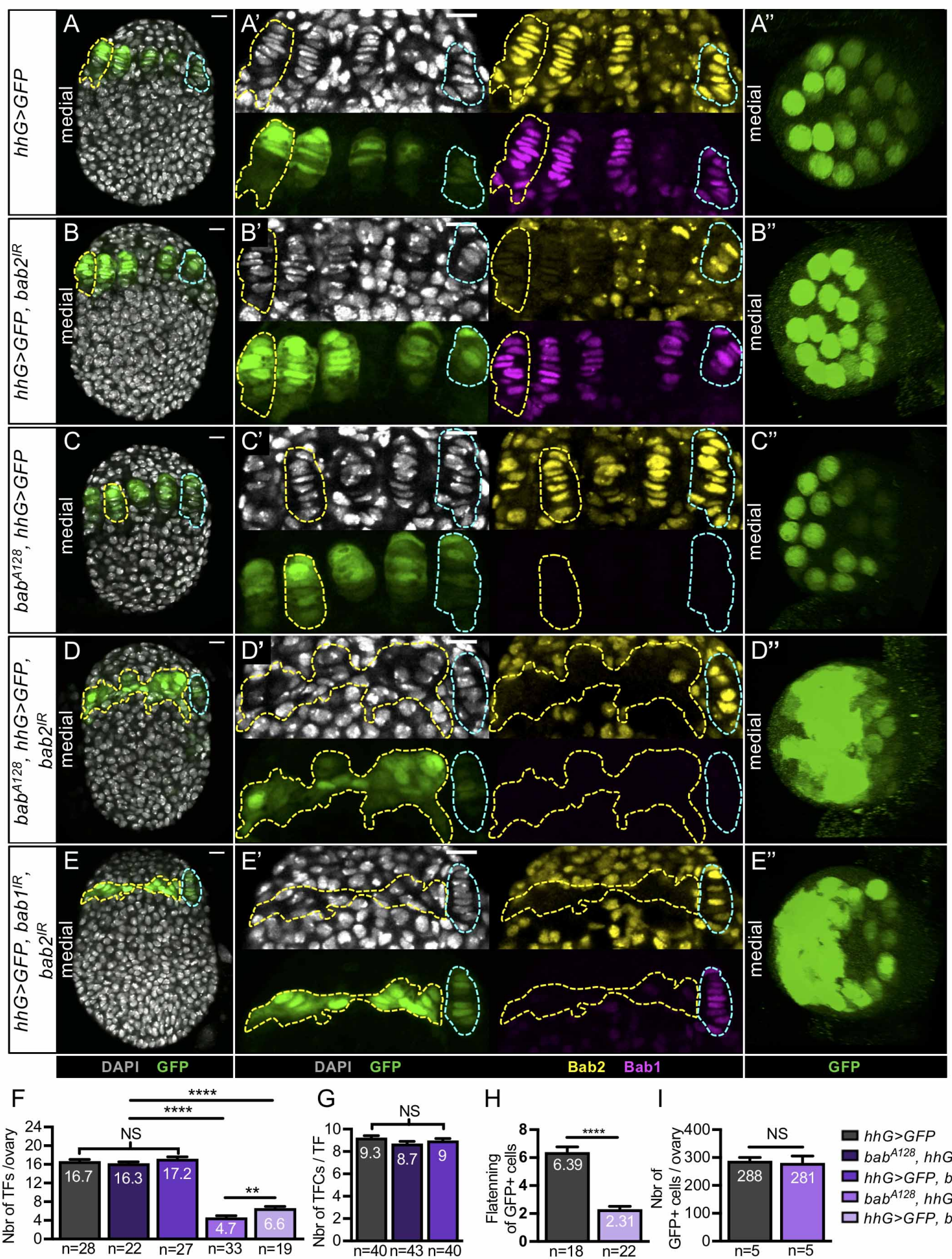

$\mathrm{H}$

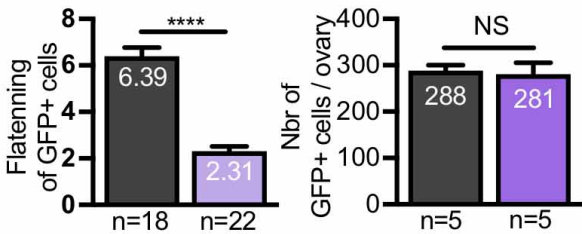

$\square h h G>G F P$

bab ${ }^{A 128}, h h G>G F P$

$\square b a b^{A 128}, h h G>G F P, b a b 2^{I R}$ $\square h h G>G F P, b a b 2^{I R}, b a b 2^{I R}$

Fig 2 

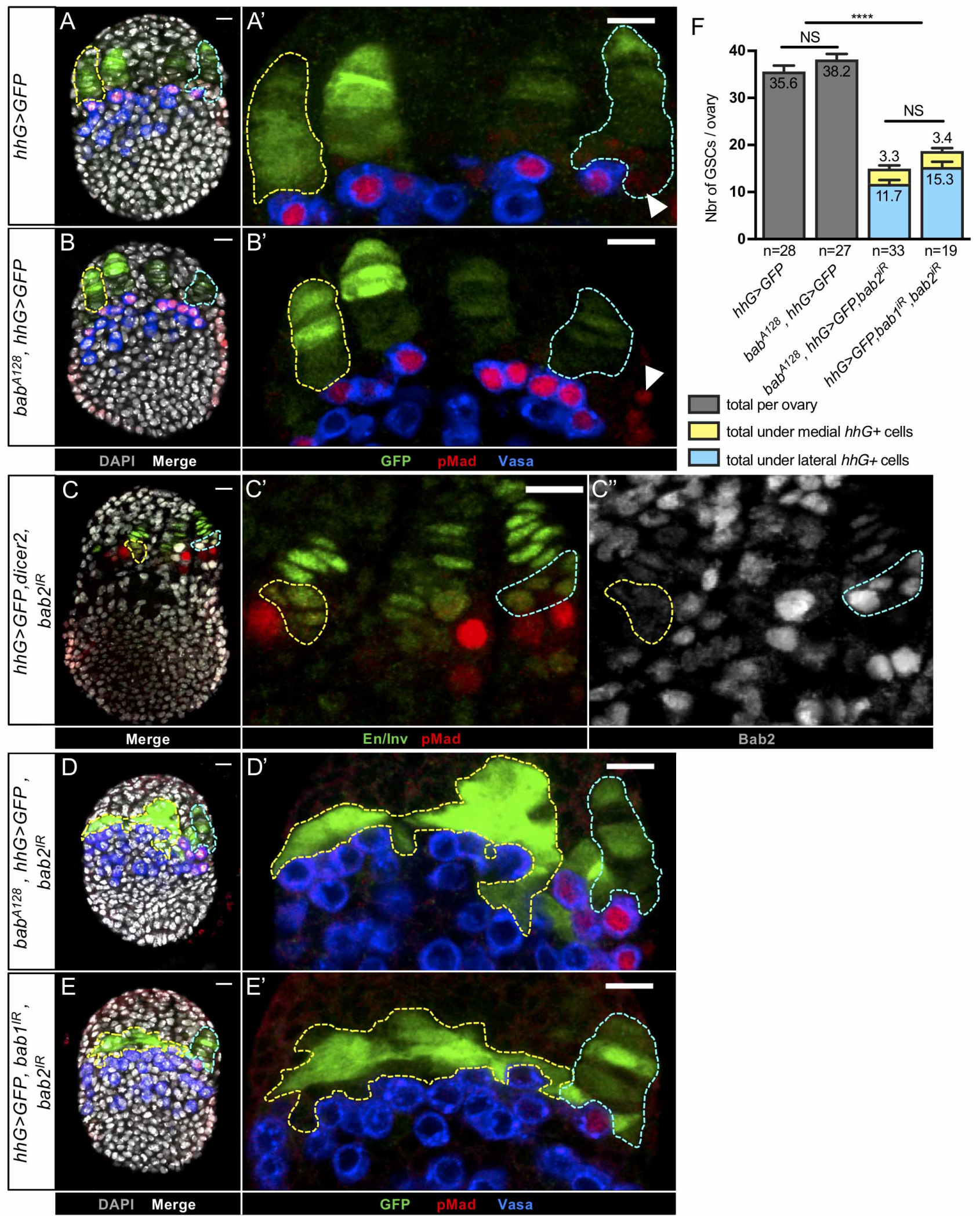

Fig 3 

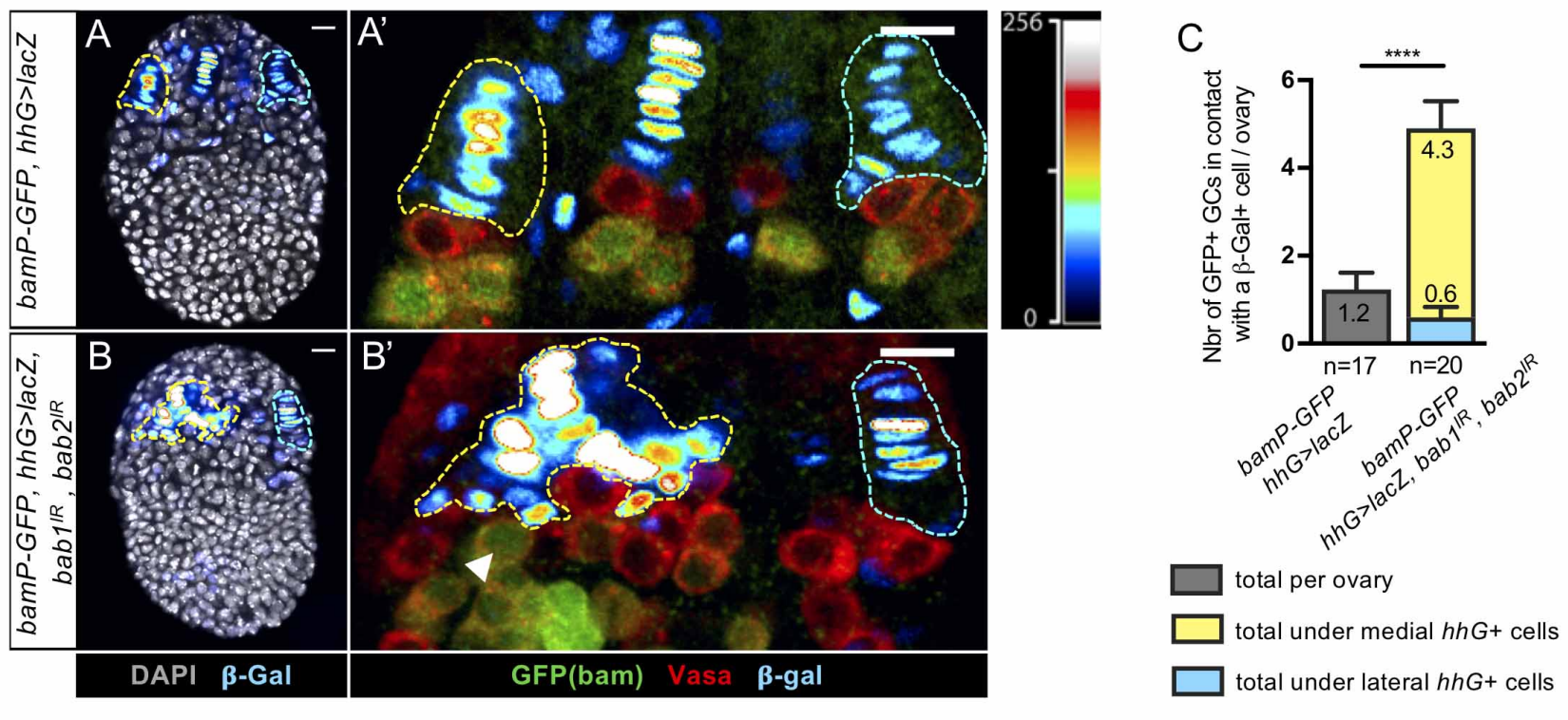

Fig 4 

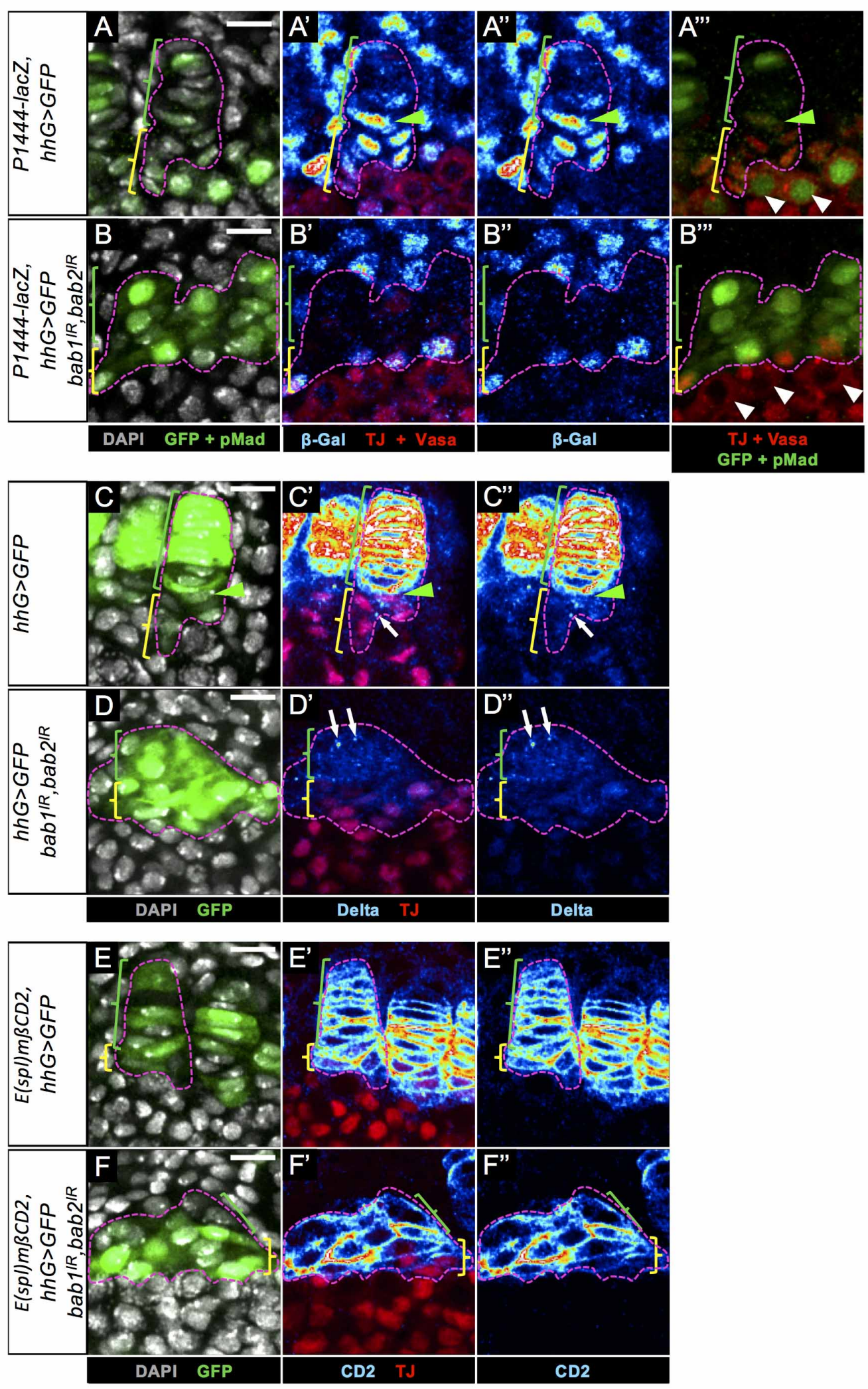

Fig 5 

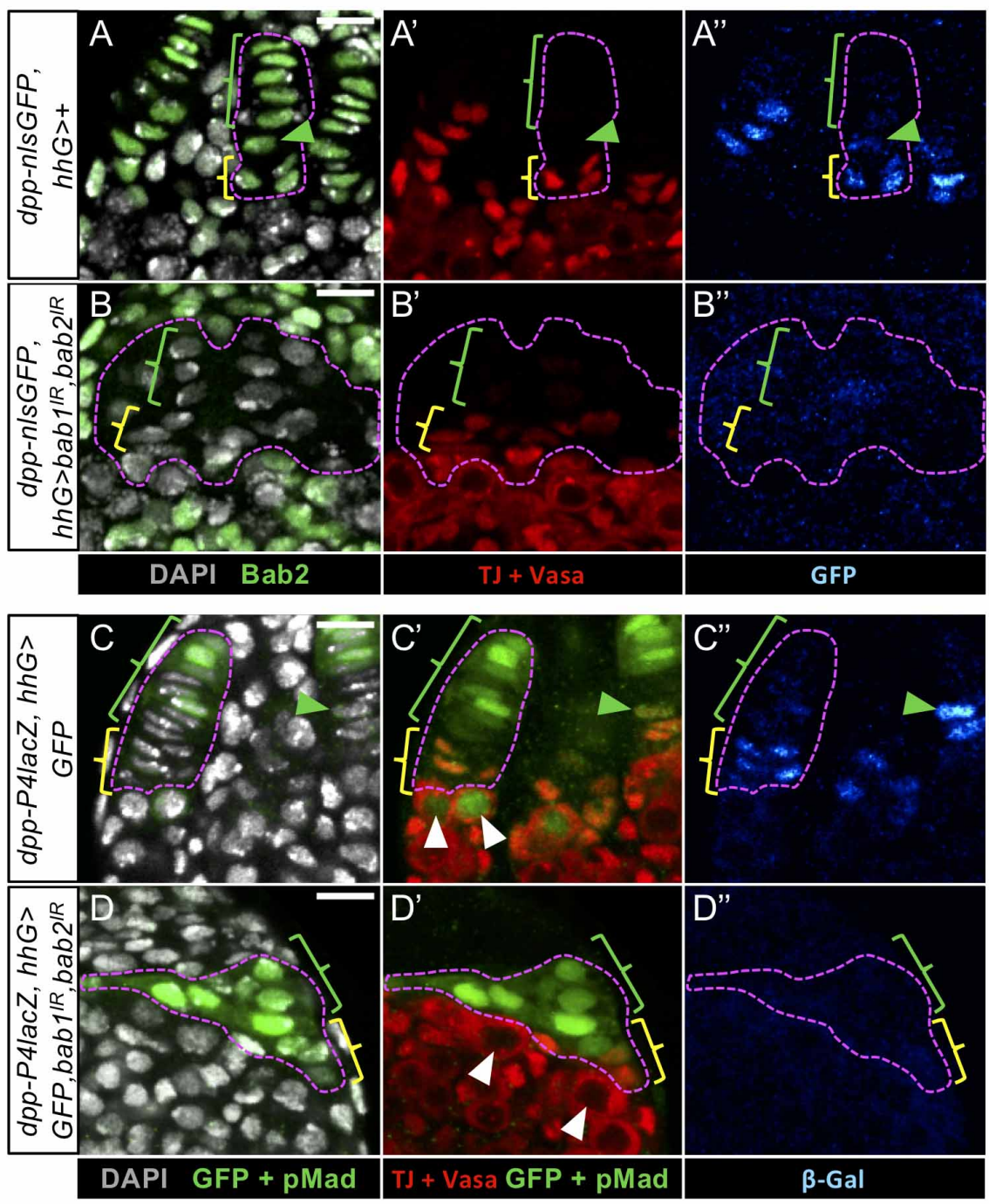

Fig 6 

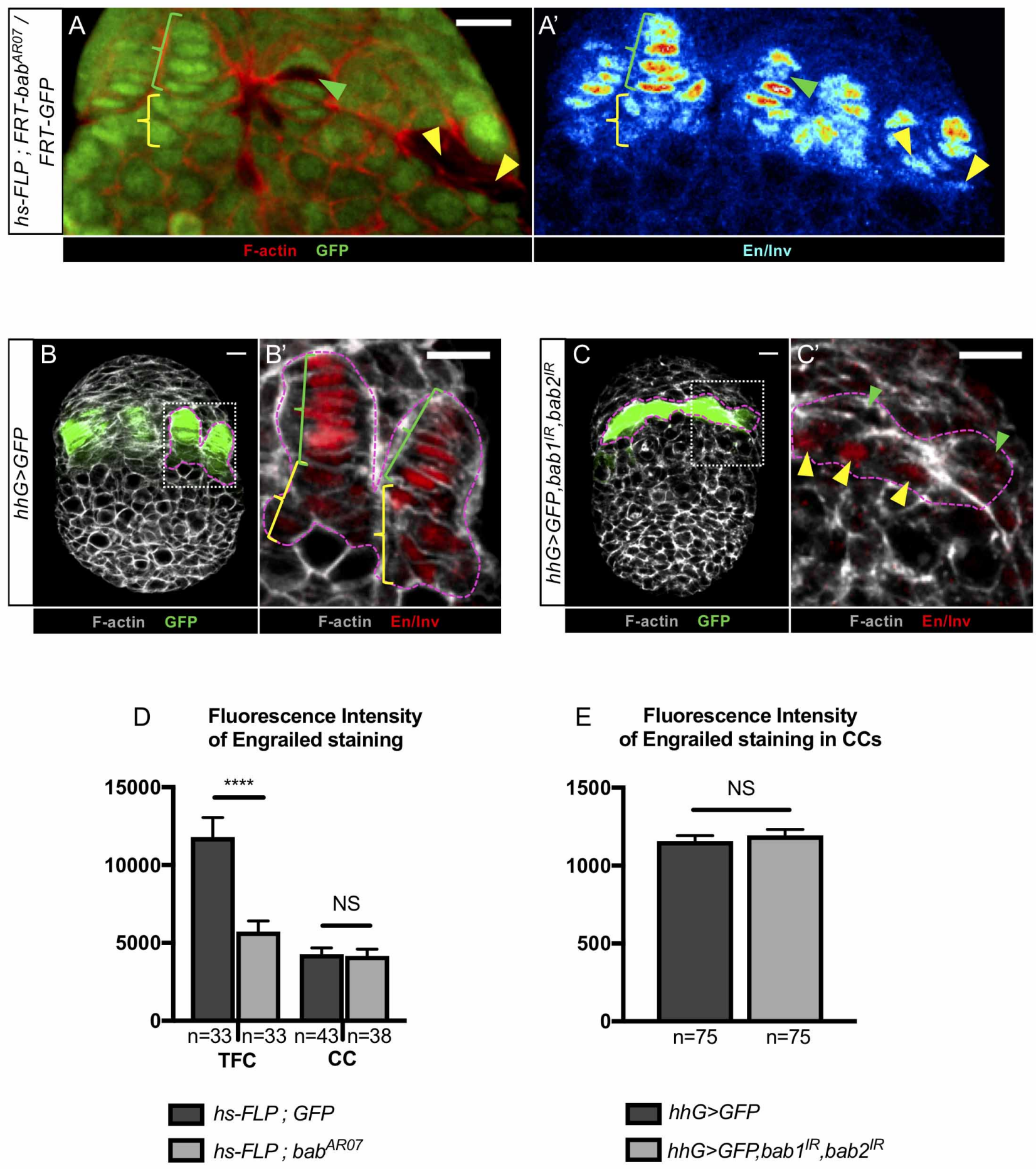

Fig 7 


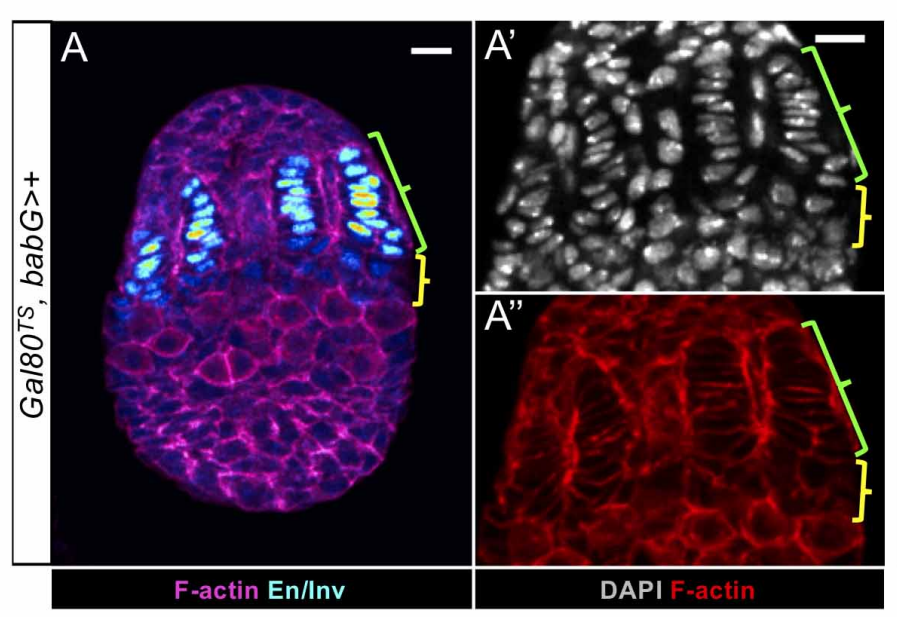

C

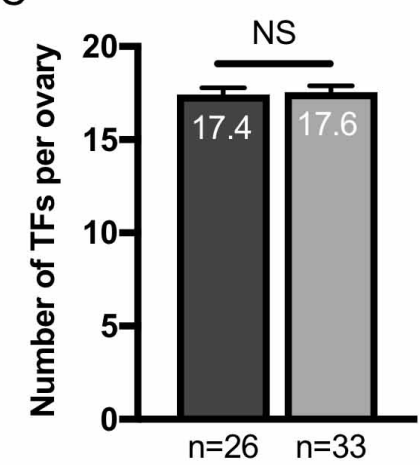

D

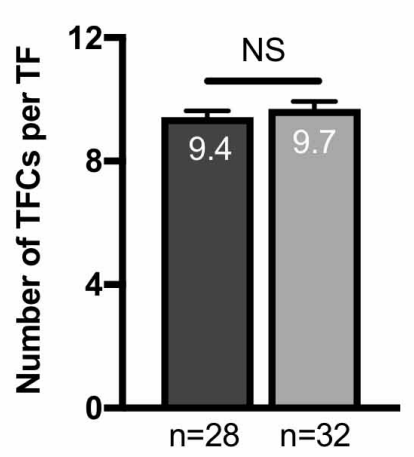

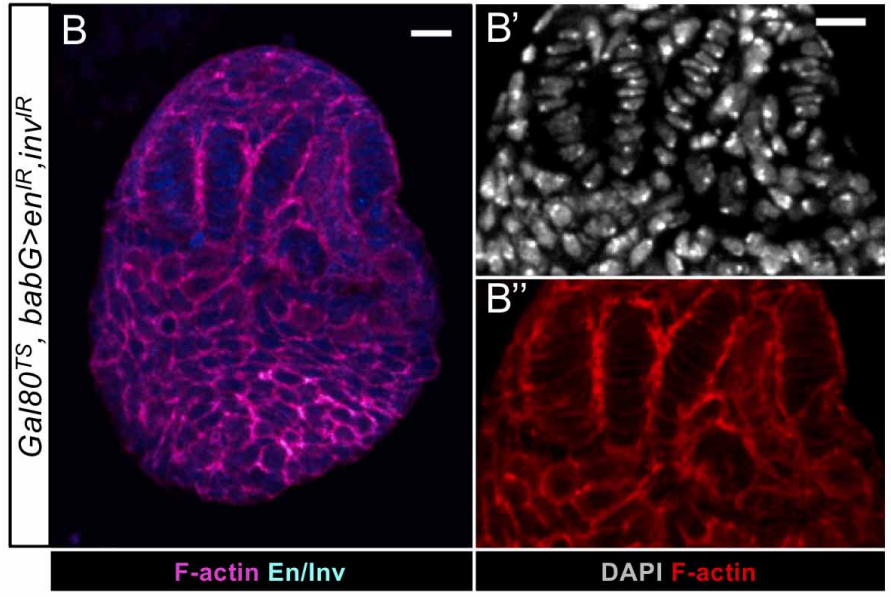

E

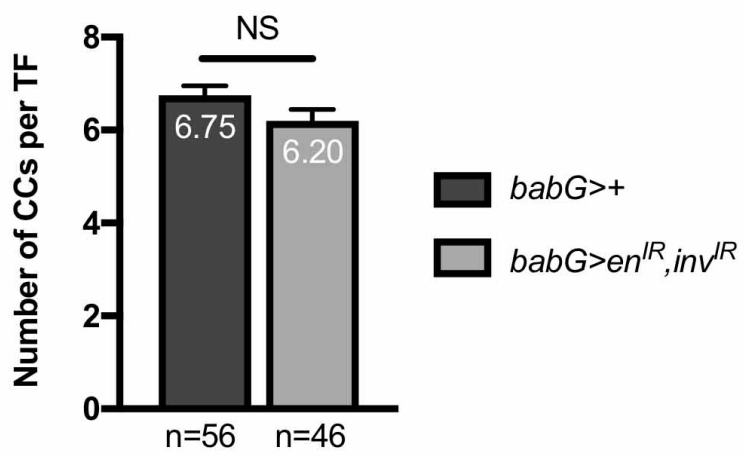

Fig 8 

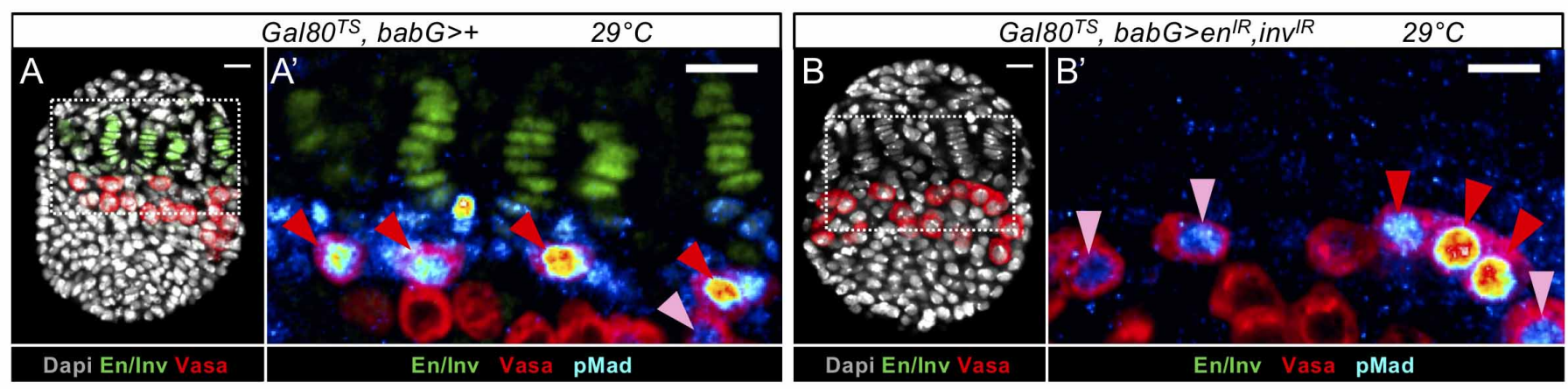

C Proportion of TF associated with:

$\square$ GSC(s) pMad + (high)
$\square$ GSC(s) pMad + (low)
$\square$ GC(s) pMad -
$\square$ no GC

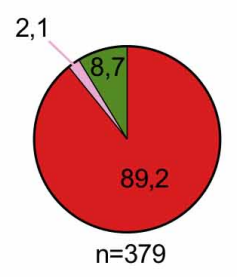

G80 $0^{T S}$ babG $>+$

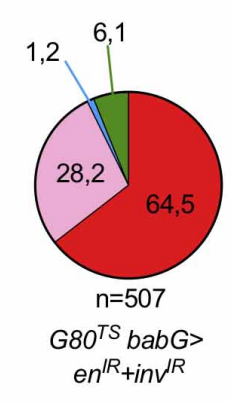

$\mathrm{D}$

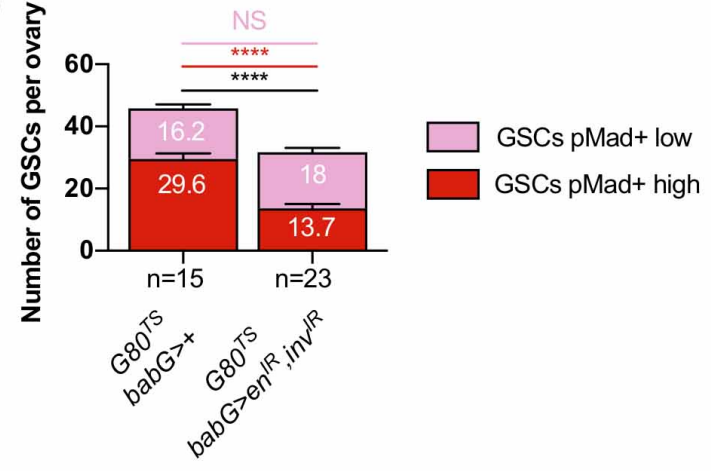

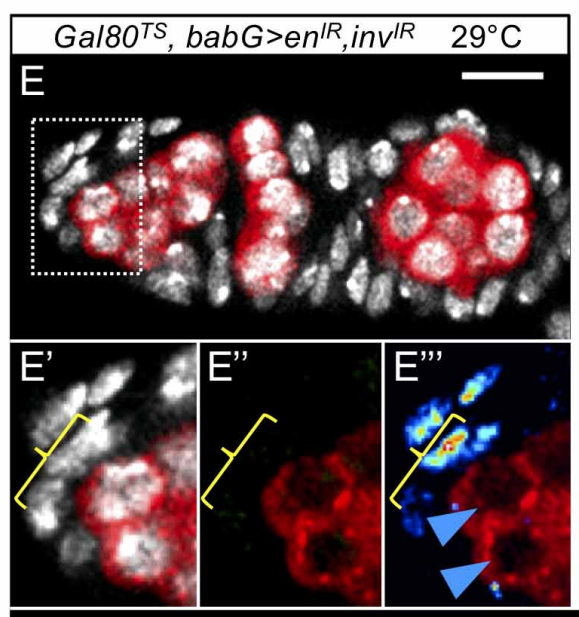
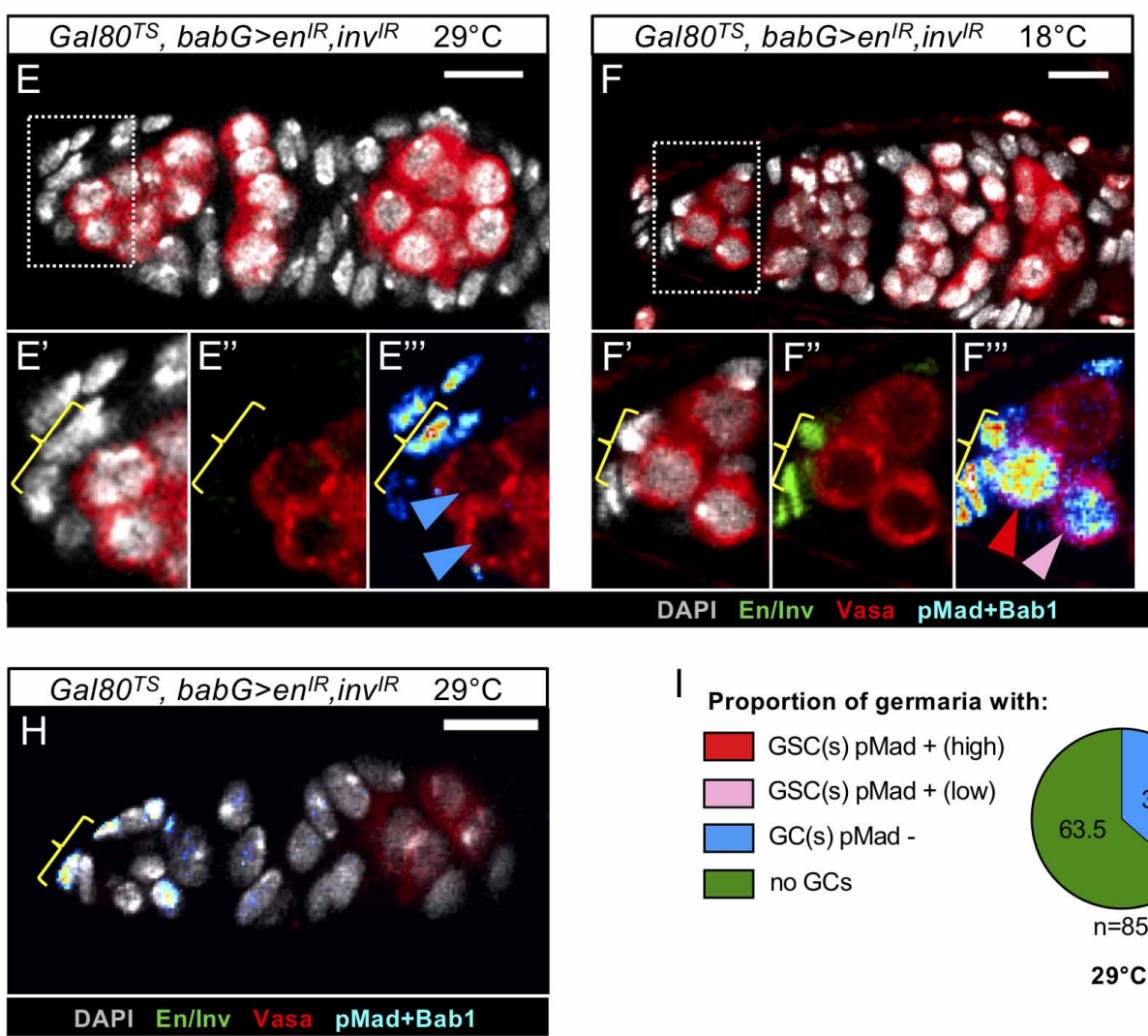

DAPI En/lnv Vasa pMad+Bab1

I Proportion of germaria with:
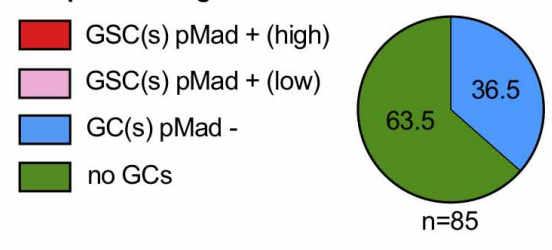

$29^{\circ} \mathrm{C}$
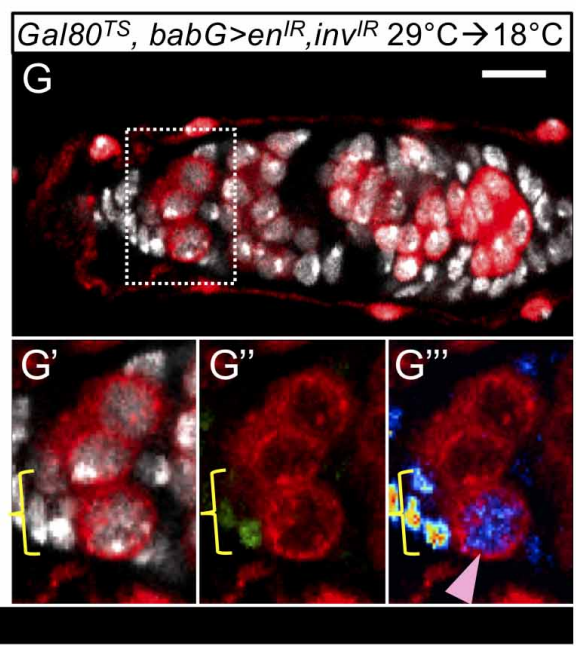

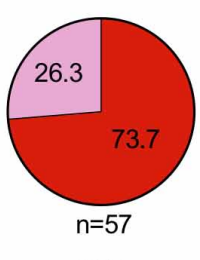

$18^{\circ} \mathrm{C}$

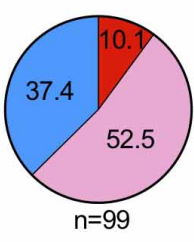

$29->18^{\circ} \mathrm{C}$

Fig 9 

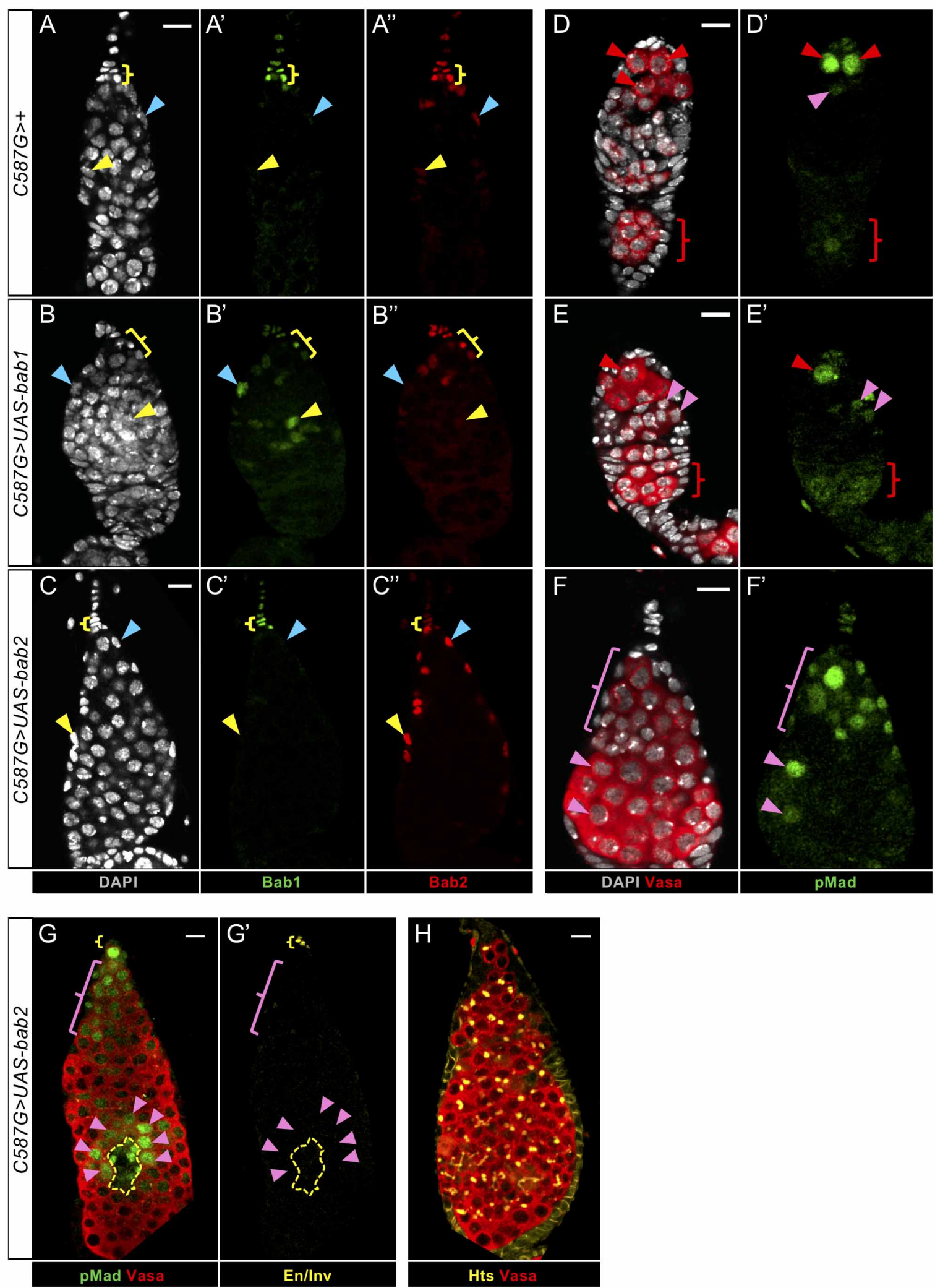

Fig 10 